\title{
EXPONENTIAL DECAY FOR A NONLINEAR MODEL FOR ELECTRICAL CONDUCTION IN BIOLOGICAL TISSUES
}

\author{
M. AMAR - D. ANDREUCCI - R. GIANNI \\ DIPARTIMENTO DI SCIENZE DI BASE E APPLICATE PER L'INGEGNERIA \\ UNIVERSITÀ DI ROMA "LA SAPIENZA" \\ VIA A. SCARPA 16, 00161 ROMA, ITALY
}

\begin{abstract}
We study the asymptotic convergence to a periodic steady state of the solution of a nonlinear system of equations with periodic boundary data modeling electrical conduction in biological tissues, both in the microscopic and in the homogenized version. Such model keeps into account the resistive behavior of the intracellular and extracellular domains and also the capacitive/resistive behavior of the lipidic cellular membrane.

The rate of convergence is analyzed and the systems of equations satisfied by the asymptotic limits are exhibited, when the resistive behavior of the membrane is described by a strictly monotone and coercive nonlinear function.

The special case of homogeneous boundary conditions is also investigated, where the coercivity assumption can be relaxed.
\end{abstract}

KEYwORDs: Asymptotic decay, stability, nonlinear homogenization, two-scale techniques, electrical impedance tomography.

AMS-MSC: 35B40, 35B27, 45K05, 92C55

\section{INTRODUCTION}

Composite materials have widespread applications in science and technology and, for this reason, have been extensively studied especially using homogenization techniques (we quote, among others, [15], [17], [19], [31], [32],[35], [36]). In this framework the authors, and co-workers, have investigated a problem arising in electric conduction in biological tissues with the purpose of obtaining some useful results for applications in electrical tomography (see [4]-[12]).

We deal with the physical problem of electric currents crossing a living tissue when an electrical potential is applied at the boundary (see [16], [18], [21], [25], [29]). Here the living tissue is regarded as a composite periodic domain made of extracellular and intracellular phases both assumed to be conductive, possibly with different conductivities, separated by a lipidic membrane experimentally found to exhibit both conductive (due to ionic channels in the membrane) and capacitive behavior. In this regard the large number of cells contained in the biological sample allows us and even imposes to use a homogenization technique. Such technique yields the system of partial differential equations satisfied by the macroscopic electric potential $u$, which is the limit of the electric potential $u_{\varepsilon}$ as the characteristic length of the cell $\varepsilon$ tends to zero. 
Clearly if we want the capacitive and the conductive behavior of the membranes to be maintained when $\varepsilon \rightarrow 0$ we must properly rescale the capacity and the conductivity of such membranes with respect to $\varepsilon$. In [8] and [12] the authors have shown that, essentially, only three scalings are physically reasonable. One of these scalings seems to be the most suitable to describe the behavior of the membranes for currents in the radiofrequency range (which is the standard frequency used in electrical tomography). In this model the magnetic field is neglected (as suggested by experimental evidence) and the potential $u_{\varepsilon}$ is assumed to satisfy an elliptic equation both in the intracellular and in the extracellular domain while on the membranes it satisfies the equation

$$
\frac{\alpha}{\varepsilon} \frac{\partial}{\partial t}\left[u_{\varepsilon}\right]+f\left(\frac{\left[u_{\varepsilon}\right]}{\varepsilon}\right)=\sigma^{\varepsilon} \nabla u_{\varepsilon} \cdot \nu_{\varepsilon}
$$

where $\left[u_{\varepsilon}\right]$ denotes the jump of the potential across the membranes and $\sigma^{\varepsilon} \nabla u_{\varepsilon} \cdot \nu_{\varepsilon}$ is the current crossing the membranes. The interface condition above was rigorously obtained in [8] by means of a concentrated capacity technique, whence the onset of the scaling specific to this model.

From a mathematical point of view the cases of linear or nonlinear $f$ are markedly different. Homogenization limits have been rigorously found in both cases. The linear case has been considered in [4], [6] and [11], via asymptotic expansion in $\varepsilon$. It has been shown that the limit potential $u$ satisfies an elliptic equation with memory for which an existence and uniqueness theorem has been proved in [5].

In the nonlinear case the approach is much more complicated and relies on the twoscale convergence technique ([2], [3], [20], [28], [34]). In this case a memory effect is still present in the limiting problem, which however does not take the form of a single partial differential equation satisfied by $u$ (see [12]). Indeed, the problem rather contains two unknowns $u$ and $u_{1}$; the latter accounts for the microscopic properties of the material and depends both on the macroscopic variable $x$ and on the microscopic variable $y$. Formally this limiting problem keeps the abstract parabolic structure which is characteristic of the microscopic scheme described above and already remarked in [9].

For a physical and biological motivation of the interest in nonlinear models of the type considered in this paper see for instance [14], [30] and [33], [1].

Going back to the technical applications of bioimpedance tomography it must be noted that usually the applied boundary potential is time harmonic, allowing for the empirical assumption that the resulting potential inside the biological material is time harmonic too. Under this assumption the behavior of the biological tissue is modeled by means of complex elliptic equations, one for every harmonic frequency. The correctness of this model has been proved by the authors in the linear case in [9], [10] and [11], investigating the time limit, as $t \rightarrow+\infty$, of the solution $u$ of the homogenized problem. There it was proved that the equations currently used in electrical tomography can be rigorously obtained by means of an asymptotic limit with respect to $t$ when time periodic boundary data are assigned. Here the asymptotic decay is exponential. As a new input, those papers revealed the relation expressing the complex admittivity of the limiting equation as an explicit function of the frequency of the boundary data and of the physical properties of the tissue. 
It is remarkable that an elliptic equation with memory in general does not exhibit asymptotic stability even if the memory kernel decreases to zero exponentially when $t \rightarrow+\infty$ (see [24]). For this reason, in the papers quoted above the result is obtained proving an asymptotic exponential convergence in $t$ for the problem of level $\varepsilon$ (i.e., before homogenization) and observing that such a convergence is stable with respect to $\varepsilon$, so that it holds true also for the limiting potential. For an alternative approach to asymptotic stability in problems with memory, relying on some extra-assumptions on the structure of the kernel see, for instance, [22] and [23]. About asymptotic decay in diffusive or electromagnetic systems see also [26], [27] and the references therein. Actually the problem at level $\varepsilon$ is not in general asymptotically stable in $t$. For instance, if $f$ is identically equal to zero, $u_{\varepsilon}$ does not tend to zero exponentially in $t$ even if a homogeneous boundary condition is assigned. Indeed such a decay requires also that the initial jumps of the potential across the cellular membranes have zero mean value on each membrane. However in the linear case we can still reduce to this setting by subtracting from the initial potential a piecewise constant function, while in the nonlinear case this is not possible and we must proceed in a different way.

We remark that such a pathology does not appear in the homogenized problem (see Remark 3.4).

Motivated by the previous considerations, in this paper we investigate the behavior as $t \rightarrow+\infty$ of the nonlinear problem introduced in [12]. We prove that if homogeneous boundary data are assigned, then the homogenized limit tends exponentially to zero for a rather general class of functions $f$; for example for functions $f$ which are Lipschitz-continuous and satisfy

$$
f\left(s_{1}\right)-f\left(s_{2}\right)>-L_{-}\left(s_{1}-s_{2}\right), \quad \forall s_{1}, s_{2} \in \boldsymbol{R} ; s_{1}>s_{2},
$$

with $L_{-}$sufficiently small (see Remark 3.5 ). Such a behavior can not be expected for solutions of the $\varepsilon$-problem, as explained above (see also Remark 3.4).

Moreover, when periodic boundary data are assigned and $f$ is coercive in the sense of (2.12) below, we obtain the exponential asymptotic convergence of the solution of the homogenized problem to a periodic solution of a suitable system of equations. Under the same assumptions an analogous result is obtained also for the solution of the $\varepsilon$-problem.

Finally, we remark that the non coercive case will be treated in [13], where a weaker form of asymptotic stability is proved.

The technical feature discriminating the cases of vanishing and general periodic data is the fact that in the latter case we are unable to rely on the technique of [9][11], which was based on eigenvalue estimates allowing us to keep into account the dissipative properties both of the intra/extra cellular phases and of the membranes. Instead, in the general case, we exploit only the coercivity of $f$.

The paper is organized as follows: in Section 2 we present the geometrical setting and the nonlinear differential model governing our problem at the microscale $\varepsilon$ as well as our main results. In Section 3 we prove the exponential decay in time of the solution of the microscopic and the macroscopic problem in the case of homogeneous boundary data, while the case of time-periodic boundary data is dealt with in Section 4. Finally, in Section 5 we prove the exponential decay in time of the solution of the homogenized 
problem, identifying also the differential system satisfied by the asymptotic limit, which is a time-periodic function.

Acknowledgments. The authors are members of the Gruppo Nazionale per l'Analisi Matematica, la Probabilità e le loro Applicazioni (GNAMPA) (M.A.) and of the Gruppo Nazionale per la Fisica Matematica (GNFM) (D.A., R.G.), of the Istituto Nazionale di Alta Matematica (INdAM).

\section{Preliminaries}

Let $\Omega$ be an open bounded subset of $\boldsymbol{R}^{N}$. In the sequel $\gamma$ or $\widetilde{\gamma}$ will denote constants which may vary from line to line and which depend on the characteristic parameters of the problem, but which are independent of $\varepsilon$ unless explicitly specified.

2.1. The geometrical setting. The typical geometry we have in mind is depicted in Figure 1. In order to be more specific, assume $N \geq 2$ and let us introduce a
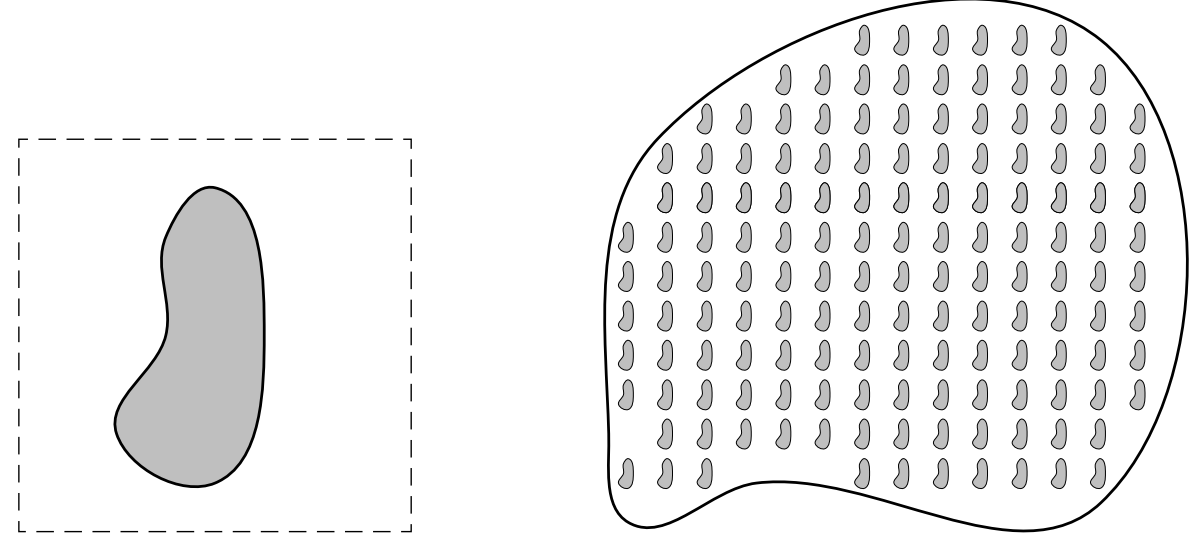

Figure 1. On the left: an example of admissible periodic unit cell $Y=$ $E_{1} \cup E_{2} \cup \Gamma$ in $\boldsymbol{R}^{2}$. Here $E_{1}$ is the shaded region and $\Gamma$ is its boundary. The remaining part of $Y$ (the white region) is $E_{2}$. On the right: the corresponding domain $\Omega=\Omega_{1}^{\varepsilon} \cup \Omega_{2}^{\varepsilon} \cup \Gamma^{\varepsilon}$. Here $\Omega_{1}^{\varepsilon}$ is the shaded region and $\Gamma^{\varepsilon}$ is its boundary. The remaining part of $\Omega$ (the white region) is $\Omega_{2}^{\varepsilon}$.

periodic open subset $E$ of $\boldsymbol{R}^{N}$, so that $E+z=E$ for all $z \in \boldsymbol{Z}^{N}$. For all $\varepsilon>0$ define $\Omega_{1}^{\varepsilon}=\Omega \cap \varepsilon E, \Omega_{2}^{\varepsilon}=\Omega \backslash \overline{\varepsilon E}$. We assume that $\Omega, E$ have regular boundary, say of class $\mathcal{C}^{\infty}$ for the sake of simplicity, and $\operatorname{dist}\left(\Gamma^{\varepsilon}, \partial \Omega\right) \geq \gamma \varepsilon$, where $\Gamma^{\varepsilon}=\partial \Omega_{1}^{\varepsilon}$. We also employ the notation $Y=(0,1)^{N}$, and $E_{1}=E \cap Y, E_{2}=Y \backslash \bar{E}, \Gamma=\partial E \cap \bar{Y}$. As a simplifying assumption, we stipulate that $E_{1}$ is a connected smooth subset of $Y$ such that $\operatorname{dist}(\Gamma, \partial Y)>0$. We denote by $\nu$ the normal unit vector to $\Gamma$ pointing into $E_{2}$, so that $\nu_{\varepsilon}(x)=\nu\left(\varepsilon^{-1} x\right)$.

For later use, we introduce also the conductivity

$$
\sigma(y)=\left\{\begin{array}{ll}
\sigma_{1} & \text { if } y \in E_{1}, \\
\sigma_{2} & \text { if } y \in E_{2},
\end{array} \quad \text { and } \quad \sigma_{0}=\left|E_{1}\right| \sigma_{1}+\left|E_{2}\right| \sigma_{2},\right.
$$


where $\sigma_{1}, \sigma_{2}$ are positive constants, and we also set $\sigma^{\varepsilon}(x)=\sigma\left(\varepsilon^{-1} x\right)$. Moreover, let us set

$$
\begin{gathered}
\mathcal{C}_{\#}^{1}(Y)=\left\{u \in \mathcal{C}^{1}(\bar{Y}) \mid u \text { is } Y \text {-periodic }\right\} \\
\mathcal{X}_{\#}^{1}(Y):=\left\{u \in L^{2}(Y) \mid u_{\mid E_{1}} \in H^{1}\left(E_{1}\right), u_{\mid E_{2}} \in H^{1}\left(E_{2}\right), \text { and } u \text { is } Y \text { - periodic }\right\},
\end{gathered}
$$

and

$$
\mathcal{X}^{1}\left(\Omega_{\varepsilon}\right):=\left\{u \in L^{2}(\Omega) \mid u_{\mid \Omega_{1}^{\varepsilon}} \in H^{1}\left(\Omega_{1}^{\varepsilon}\right), u_{\mid \Omega_{2}^{\varepsilon}} \in H^{1}\left(\Omega_{2}^{\varepsilon}\right)\right\} .
$$

We note that, if $u \in \mathcal{X}_{\#}^{1}(Y)$ then the traces of $u_{\mid E_{i}}$ on $\Gamma$, for $i=1,2$, belong to $H^{1 / 2}(\Gamma)$, as well as $u \in \mathcal{X}^{1}\left(\Omega_{\varepsilon}\right)$ implies that the traces of $u_{\mid \Omega_{i}^{\varepsilon}}$ on $\Gamma^{\varepsilon}$, for $i=1,2$, belong to $H^{1 / 2}\left(\Gamma^{\varepsilon}\right)$.

2.2. Statement of the problem. We consider the model problem

$$
\begin{aligned}
-\operatorname{div}\left(\sigma_{1} \nabla u_{\varepsilon}\right) & =0, & & \text { in } \Omega_{1}^{\varepsilon} \times(0, T) ; \\
-\operatorname{div}\left(\sigma_{2} \nabla u_{\varepsilon}\right) & =0, & & \text { in } \Omega_{2}^{\varepsilon} \times(0, T) ; \\
{\left[\sigma^{\varepsilon} \nabla u_{\varepsilon} \cdot \nu_{\varepsilon}\right] } & =0, & & \text { on } \Gamma^{\varepsilon} \times(0, T) ; \\
\frac{\alpha}{\varepsilon} \frac{\partial}{\partial t}\left[u_{\varepsilon}\right]+f\left(\frac{\left[u_{\varepsilon}\right]}{\varepsilon}\right) & =\sigma^{\varepsilon} \nabla u_{\varepsilon} \cdot \nu_{\varepsilon}, & & \text { on } \Gamma^{\varepsilon} \times(0, T) ; \\
{\left[u_{\varepsilon}\right](x, 0) } & =S_{\varepsilon}(x), & & \text { on } \Gamma^{\varepsilon} ; \\
u_{\varepsilon}(x) & =\Psi(x, t), & & \text { on } \partial \Omega \times(0, T),
\end{aligned}
$$

where $\sigma_{1}, \sigma_{2}$ are defined in the previous subsection and $\alpha>0$ is a constant. We note that, by its definition, $\nu_{\varepsilon}$ is the normal unit vector to $\Gamma^{\varepsilon}$ pointing into $\Omega_{2}^{\varepsilon}$. Since $u_{\varepsilon}$ is not in general continuous across $\Gamma^{\varepsilon}$ we set

$$
\begin{gathered}
u_{\varepsilon}^{(1)}:=\text { trace of } u_{\varepsilon \mid \Omega_{1}^{\varepsilon}} \text { on } \Gamma^{\varepsilon} \times(0, T) ; \quad u_{\varepsilon}^{(2)}:=\text { trace of } u_{\varepsilon \mid \Omega_{2}^{\varepsilon}} \text { on } \Gamma^{\varepsilon} \times(0, T) ; \\
{\left[u_{\varepsilon}\right]:=u_{\varepsilon}^{(2)}-u_{\varepsilon}^{(1)} .}
\end{gathered}
$$

Similar conventions are employed for other quantities, for example in (2.3). In this framework we will assume that

$$
S_{\varepsilon} \in H^{1 / 2}\left(\Gamma^{\varepsilon}\right), \quad \int_{\Gamma^{\varepsilon}} S_{\varepsilon}^{2}(x) \mathrm{d} \sigma \leq \gamma \varepsilon,
$$

motivated by (3.1) below. The function $f: \boldsymbol{R} \rightarrow \boldsymbol{R}$ is assumed to satisfy

i) $f$ is a Lipschitz-continuous function with Lipschitz constant $L$,

ii) $f(0)=0$.

In particular, denoting by $L_{-}, L_{+}>0$ the Lipschitz constants of $f$ from below and from above respectively, we can write

$$
-L_{-}\left(s_{1}-s_{2}\right) \leq f\left(s_{1}\right)-f\left(s_{2}\right) \leq L_{+}\left(s_{1}-s_{2}\right), \quad \forall s_{1}, s_{2} \in \boldsymbol{R} \quad \text { with } s_{1}>s_{2} .
$$

The first inequality above, together with (2.8), implies also that

$$
f(s) s \geq-L_{-} s^{2}, \quad \forall s \in \boldsymbol{R} .
$$


Finally, $\Psi: \Omega \times \boldsymbol{R} \rightarrow \boldsymbol{R}$ is a function satisfying the following assumptions

$$
\begin{array}{ll}
\text { i) } & \Psi \in L_{l o c}^{2}\left(\boldsymbol{R} ; H^{2}(\Omega)\right) ; \\
\text { ii) } & \Psi_{t} \in L_{l o c}^{2}\left(\boldsymbol{R} ; H^{1}(\Omega)\right) ; \\
\text { iii }) & \Psi(x, \cdot) \text { is 1-periodic for a.e. } x \in \Omega .
\end{array}
$$

The regularity in (2.10) will be used in Lemma 4.1 and 5.2.

The set of equations (2.1)-(2.6) models electrical conduction in a biological tissue. Recalling the discussion in the Introduction, we notice that the first term in the left hand side of (2.4) models the behavior of the lipidic cell membrane which acts mainly as a capacitor, while the second term in the left hand side keeps into account the resistive behavior of the membrane which is caused by channels allowing charged molecules to go through. Here the resistive behavior is assumed to be nonlinear and it is relevant that the small parameter $\varepsilon$, which is of the order of magnitude of the cell width, appears inside the argument of $f$. Existence of solutions to problem (2.1)(2.6) has been proved in [7] to which we refer for the rigorous definition of (weak) solution.

Our first result concerns the case of homogeneous boundary data.

Theorem 2.1. For every $\varepsilon>0$, let $u_{\varepsilon}$ be the solution of problem (2.1)-(2.6) with $\Psi \equiv 0$. Then, if $L_{-}$is sufficiently small (depending on the constants $\sigma_{1}, \sigma_{2}, \alpha$ and the geometry of $\Gamma$ ), we have

$$
\left\|u_{\varepsilon}(\cdot, t)\right\|_{L^{2}(\Omega)}^{2} \leq \gamma\left(\mathrm{e}^{-\beta t}+\varepsilon^{2} \mathrm{e}^{\frac{2 L_{-}}{\alpha} t}\right) \quad \forall t>1,
$$

where $\beta, \gamma>0$ are constants independent from $\varepsilon$.

In the following we'll need a more stringent assumption on $f$, that is

$$
f \in \mathcal{C}^{1}(\boldsymbol{R}), \quad f^{\prime}(s) \geq \kappa>0, \quad \forall s \in \boldsymbol{R},
$$

for a suitable $\kappa>0$.

We also introduce a time-periodic version of the problem solved by $u_{\varepsilon}$, i.e.,

$$
\begin{array}{rlrl}
-\operatorname{div}\left(\sigma^{\varepsilon} \nabla u_{\varepsilon}^{\#}\right) & =0, & & \text { in }\left(\Omega_{1}^{\varepsilon} \cup \Omega_{2}^{\varepsilon}\right) \times \boldsymbol{R} ; \\
{\left[\sigma^{\varepsilon} \nabla u_{\varepsilon}^{\#} \cdot \nu_{\varepsilon}\right]} & =0, & & \text { on } \Gamma^{\varepsilon} \times \boldsymbol{R} ; \\
\frac{\alpha}{\varepsilon} \frac{\partial}{\partial t}\left[u_{\varepsilon}^{\#}\right]+f\left(\frac{\left[u_{\varepsilon}^{\#}\right]}{\varepsilon}\right) & =\left(\sigma^{\varepsilon} \nabla u_{\varepsilon}^{\#} \cdot \nu_{\varepsilon}\right), & & \text { on } \Gamma^{\varepsilon} \times \boldsymbol{R} ; \\
u_{\varepsilon}^{\#}(x, t) & =\Psi(x, t), & & \text { on } \partial \Omega \times \boldsymbol{R} ; \\
u_{\varepsilon}^{\#}(x, \cdot) \text { is 1-periodic, }, & & \text { in } \Omega .
\end{array}
$$

Indeed, this problem is derived from (2.1)-(2.6) by replacing equation (2.5) with (2.17). In Definition 4.13 we introduce the notion of periodic (weak) solution.

Theorem 2.2. Let $\varepsilon>0$ be fixed and let $u_{\varepsilon}$, respectively $u_{\varepsilon}^{\#}$, be the solution of problem (2.1)-(2.6), respectively of problem (2.13)-(2.17). Assume (2.12). Then, for $t \rightarrow+\infty, u_{\varepsilon} \rightarrow u_{\varepsilon}^{\#}$ in the following sense: there exist $\beta=2 \kappa / \alpha$ and $\gamma>0$, 
independent of $\varepsilon$, such that

$$
\begin{array}{lr}
\left\|u_{\varepsilon}(\cdot, t)-u_{\varepsilon}^{\#}(\cdot, t)\right\|_{L^{2}(\Omega)} \leq \gamma \mathrm{e}^{-\beta t / 2} ; & \forall t>1 ; \\
\left\|\nabla u_{\varepsilon}(\cdot, t)-\nabla u_{\varepsilon}^{\#}(\cdot, t)\right\|_{L^{2}\left(\Omega_{i}^{\varepsilon}\right)} \leq \gamma \mathrm{e}^{-\beta t / 2} ; & \forall t>1 ; i=1,2 ; \\
\frac{1}{\sqrt{\varepsilon}}\left\|\left[u_{\varepsilon}\right](\cdot, t)-\left[u_{\varepsilon}^{\#}\right](\cdot, t)\right\|_{L^{2}\left(\Gamma^{\varepsilon}\right)} \leq \gamma \mathrm{e}^{-\beta t / 2} ; & \forall t>1 .
\end{array}
$$

It has been shown in [12] that as $\varepsilon \rightarrow 0$ the sequence $u_{\varepsilon}$ converges in the sense of two-scales (see also Section 5) to the solution $\left(u, u^{1}\right), u=u(x, t), u^{1}=u^{1}(x, y, t)$, as defined in Definition 5.1, of the problem

$$
\begin{aligned}
-\operatorname{div}\left(\sigma_{0} \nabla u+\int_{Y} \sigma \nabla_{y} u^{1} \mathrm{~d} y\right) & =0, & & \text { in } \Omega \times(0, T) ; \\
-\operatorname{div}_{y}\left(\sigma \nabla u+\sigma \nabla_{y} u^{1}\right) & =0, & & \text { in } \Omega \times\left(E_{1} \cup E_{2}\right) \times(0, T) ; \\
{\left[\sigma\left(\nabla u+\nabla_{y} u^{1}\right) \cdot \nu\right] } & =0, & & \text { on } \Omega \times \Gamma \times(0, T) ; \\
\alpha \frac{\partial}{\partial t}\left[u^{1}\right]+f\left(\left[u^{1}\right]\right) & =\sigma\left(\nabla u+\nabla_{y} u^{1}\right) \cdot \nu, & & \text { on } \Omega \times \Gamma \times(0, T) ; \\
{\left[u^{1}\right](x, y, 0) } & =S_{1}(x, y), & & \text { on } \Omega \times \Gamma ; \\
u(x, t) & =\Psi(x, t), & & \text { on } \partial \Omega \times(0, T) .
\end{aligned}
$$

In order to obtain this homogenization result we have to assume that the initial data $S_{\varepsilon}$ satisfy the additional requirements

$$
S_{\varepsilon} / \varepsilon \text { two-scale converges in } L^{2}\left(\Omega ; L^{2}(\Gamma)\right) \text { to } S_{1}
$$

where $S_{1}$ is such that $S_{1}(x, \cdot)=S_{\mid \Gamma}(x, \cdot)$ for some $S \in \mathcal{C}\left(\bar{\Omega} ; \mathcal{C}_{\#}^{1}(Y)\right)$, and

$$
\lim _{\varepsilon \rightarrow 0} \varepsilon \int_{\Gamma^{\varepsilon}}\left(\frac{S_{\varepsilon}}{\varepsilon}\right)^{2}(x) \mathrm{d} \sigma=\int_{\Omega} \int_{\Gamma} S_{1}^{2}(x, y) \mathrm{d} x \mathrm{~d} \sigma(y) .
$$

Remark 2.3. Estimates (2.18)-(2.20) are uniform with respect to $\varepsilon$; but in order to be able to pass to the limit $\varepsilon \rightarrow 0$ in them a homogenization result for the periodic problem should be available. In [12] however the proof was given for the initial value problem and hinged on the property of the initial data mentioned above, which should now be circumvented.

Here we prefer to develop a different approach to asymptotics that can be applied to more general two-scale problems, not necessarily originating as homogenization limits; see Remark 5.6. 
We introduce the following periodic version of problem (2.21)-(2.26):

$$
\begin{array}{rlrl}
-\operatorname{div}\left(\sigma_{0} \nabla u^{\#}+\int_{Y} \sigma \nabla_{y} u^{1, \#} \mathrm{~d} y\right) & =0, & & \text { in } \Omega \times \boldsymbol{R} ; \\
-\operatorname{div}_{y}\left(\sigma \nabla u^{\#}+\sigma \nabla_{y} u^{1, \#}\right) & =0, & & \text { in } \Omega \times\left(E_{1} \cup E_{2}\right) \times \boldsymbol{R} ; \\
{\left[\sigma\left(\nabla u^{\#}+\nabla_{y} u^{1, \#}\right) \cdot \nu\right]} & =0, & & \text { on } \Omega \times \Gamma \times \boldsymbol{R} ; \\
\alpha \frac{\partial}{\partial t}\left[u^{1, \#}\right]+f\left(\left[u^{1, \#}\right]\right) & =\sigma\left(\nabla u^{\#}+\nabla_{y} u^{1, \#}\right) \cdot \nu & \text { on } \Omega \times \Gamma \times \boldsymbol{R} ; \\
{\left[u^{1, \#}\right](x, y, \cdot)} & \text { is } 1 \text {-periodic, } & & \text { on } \Omega \times \Gamma ; \\
u^{\#}(x, t) & =\Psi(x, t), & & \text { on } \partial \Omega \times \boldsymbol{R} .
\end{array}
$$

For this problem we have

Theorem 2.4. Problem (2.29)-(2.34) has a unique solution in the sense of Definition 5.7 .

Finally we prove the following convergence result.

Theorem 2.5. With the notation above, and assuming (2.12), (2.27) and (2.28), we have for all $t>1$

$$
\begin{aligned}
& \left\|u(\cdot, t)-u^{\#}(\cdot, t)\right\|_{H^{1}(\Omega)} \leq \gamma \mathrm{e}^{-\beta t / 2} \\
& \left\|u^{1}(\cdot, t)-u^{1, \#}(\cdot, t)\right\|_{L^{2}(Y)}+\left\|\nabla u^{1}(\cdot, t)-\nabla u^{1, \#}(\cdot, t)\right\|_{L^{2}(Y)} \leq \gamma \mathrm{e}^{-\beta t / 2} \\
& \left\|\left[u^{1}\right](\cdot, t)-\left[u^{1, \#}\right](\cdot, t)\right\|_{L^{2}(\Gamma)} \leq \gamma \mathrm{e}^{-\beta t / 2}
\end{aligned}
$$

for suitable $\beta, \gamma>0$.

\section{Exponential DeCAY of the SOlution of the $\varepsilon$-PROBlem}

The purpose of this section is to prove the asymptotic decay as $t \rightarrow+\infty$ of the solution to (2.1)-(2.6) with homogeneous boundary data $\Psi \equiv 0$, that is Theorem 2.1. Let us recall that, under our assumptions, the solution $u_{\varepsilon}$ of $(2.1)-(2.6)$ satisfies for $0<t<T$ the following energy inequality (easily obtained on multiplying (2.1) and (2.2) by $u_{\varepsilon}$ and integrating formally by parts; see also [7])

$$
\begin{aligned}
\int_{0}^{t} \int_{\Omega} \sigma^{\varepsilon}\left|\nabla u_{\varepsilon}\right|^{2} \mathrm{~d} x \mathrm{~d} \tau+\frac{\alpha}{2 \varepsilon} \int_{\Gamma^{\varepsilon}}\left[u_{\varepsilon}\right]^{2}(x, t) \mathrm{d} \sigma+\int_{0}^{t} \int_{\Gamma^{\varepsilon}} f & \left(\frac{\left[u_{\varepsilon}\right]}{\varepsilon}\right)\left[u_{\varepsilon}\right] \mathrm{d} \sigma \mathrm{d} \tau \\
& =\frac{\alpha}{2 \varepsilon} \int_{\Gamma^{\varepsilon}} S_{\varepsilon}^{2}(x) \mathrm{d} \sigma \leq \frac{\alpha}{2} \gamma .
\end{aligned}
$$

If $f$ is monotone increasing (3.1) yields

$$
\int_{0}^{T} \int_{\Omega} \sigma^{\varepsilon}\left|\nabla u_{\varepsilon}\right|^{2} \mathrm{~d} x \mathrm{~d} \tau+\sup _{t \in[0, T]} \frac{\alpha}{2 \varepsilon} \int_{\Gamma^{\varepsilon}}\left[u_{\varepsilon}\right]^{2}(x, t) \mathrm{d} \sigma \leq \gamma .
$$


In general, using Gronwall lemma, we get

$$
\int_{0}^{T} \int_{\Omega} \sigma^{\varepsilon}\left|\nabla u_{\varepsilon}\right|^{2} \mathrm{~d} x \mathrm{~d} \tau+\sup _{t \in[0, T]} \frac{\alpha}{2 \varepsilon} \int_{\Gamma^{\varepsilon}}\left[u_{\varepsilon}\right]^{2}(x, t) \mathrm{d} \sigma \leq \gamma \mathrm{e}^{\frac{2 L_{-}}{\alpha} T} .
$$

From now on we will set $\Gamma_{k}^{\varepsilon}=\varepsilon \Gamma^{k}:=\varepsilon\left(\Gamma+\mathbf{z}_{k}\right)$ and $\Omega_{1, k}^{\varepsilon}=\varepsilon E_{1}^{k}:=\varepsilon\left(E_{1}+\mathbf{z}_{k}\right)$ for $k \in \mathcal{I}$, where $\mathbf{z}_{k} \in \boldsymbol{Z}^{N}$ and $\mathcal{I}$ is a suitable finite set of indexes covering all the components of $\Gamma^{\varepsilon}$. Moreover, we introduce the function $w_{\varepsilon}(x, t)$ given by

$$
w_{\varepsilon}(x, t)= \begin{cases}0 & \text { if }(x, t) \in \Omega_{2}^{\varepsilon} \times(0, T) ; \\ -\frac{1}{\left|\Gamma_{k}^{\varepsilon}\right|} \int_{\Gamma_{k}^{\varepsilon}}\left[u_{\varepsilon}\right] \mathrm{d} \sigma & \text { if }(x, t) \in \Omega_{1, k}^{\varepsilon} \times(0, T), k \in \mathcal{I} .\end{cases}
$$

Notice that $\left|\Gamma_{k}^{\varepsilon}\right|=\varepsilon^{N-1}|\Gamma|$ and that the piecewise constant function $w_{\varepsilon}$ solves a rather trivial analogue of (2.1)-(2.6), whence we single out the interface condition

$$
\frac{\alpha}{\varepsilon} \frac{\partial}{\partial t}\left[w_{\varepsilon}\right]=-\frac{1}{\left|\Gamma_{k}^{\varepsilon}\right|} \int_{\Gamma_{k}^{\varepsilon}} f\left(\frac{\left[u_{\varepsilon}\right]}{\varepsilon}\right) \mathrm{d} \sigma, \quad \text { on } \Gamma_{k}^{\varepsilon} \times(0, T) ; k \in \mathcal{I},
$$

following from integrating over $\Gamma_{k}^{\varepsilon}$ equation (2.4) and recalling that

$$
\int_{\Gamma_{k}^{\varepsilon}} \sigma^{\varepsilon} \nabla u_{\varepsilon} \cdot \nu_{\varepsilon} \mathrm{d} \sigma=0
$$

because of $(2.1)-(2.2)$. Finally, we set $q_{\varepsilon}=u_{\varepsilon}-w_{\varepsilon}$; it is immediately seen that $q_{\varepsilon}$ solves

$$
\begin{aligned}
-\operatorname{div}\left(\sigma_{1} \nabla q_{\varepsilon}\right) & =0, & & \text { in } \Omega_{1}^{\varepsilon} \times(0, T) ; \\
-\operatorname{div}\left(\sigma_{2} \nabla q_{\varepsilon}\right) & =0, & & \text { in } \Omega_{2}^{\varepsilon} \times(0, T) ; \\
{\left[\sigma^{\varepsilon} \nabla q_{\varepsilon} \cdot \nu_{\varepsilon}\right] } & =0, & & \text { on } \Gamma^{\varepsilon} \times(0, T) ; \\
\frac{\alpha}{\varepsilon} \frac{\partial}{\partial t}\left[q_{\varepsilon}\right]+f\left(\frac{\left[q_{\varepsilon}\right]}{\varepsilon}+\frac{\left[w_{\varepsilon}\right]}{\varepsilon}\right) & =\frac{1}{\left|\Gamma_{k}^{\varepsilon}\right|} \int_{\Gamma_{k}^{\varepsilon}} f\left(\frac{\left[u_{\varepsilon}\right]}{\varepsilon}\right) \mathrm{d} \sigma+\sigma^{\varepsilon} \nabla q_{\varepsilon} \cdot \nu_{\varepsilon}, & & \text { on } \Gamma_{k}^{\varepsilon} \times(0, T) ; k \in \mathcal{I} ; \\
{\left[q_{\varepsilon}\right](x, 0) } & =S_{\varepsilon}(x)-\frac{1}{\left|\Gamma_{k}^{\varepsilon}\right|} \int_{\Gamma_{k}^{\varepsilon}} S_{\varepsilon}(x) \mathrm{d} \sigma, & & \text { on } \Gamma_{k}^{\varepsilon} ; k \in \mathcal{I} ; \\
q_{\varepsilon}(x) & =0, & & \text { on } \partial \Omega \times(0, T) .
\end{aligned}
$$

Moreover, on integrating on $\Gamma_{k}^{\varepsilon}$ equation (3.9) and taking into account that

$$
\int_{\Gamma_{k}^{\varepsilon}} \sigma^{\varepsilon} \nabla q_{\varepsilon} \cdot \nu_{\varepsilon} \mathrm{d} \sigma=0
$$

it follows that

$$
\frac{\alpha}{\varepsilon} \frac{d}{d t} \int_{\Gamma_{k}^{\varepsilon}}\left[q_{\varepsilon}\right] \mathrm{d} \sigma=0, \quad k \in \mathcal{I}
$$


so that also using (3.10) we have

$$
\int_{\Gamma_{k}^{\varepsilon}}\left[q_{\varepsilon}(x, t)\right] \mathrm{d} \sigma(x)=0, \quad \int_{\Gamma_{k}^{\varepsilon}}\left[q_{\varepsilon, t}(x, t)\right] \mathrm{d} \sigma(x)=0, \quad t \in[0, T), k \in \mathcal{I} .
$$

We write equation (3.9) in the more convenient form

$$
\frac{\alpha}{\varepsilon} \frac{\partial}{\partial t}\left[q_{\varepsilon}\right]+f\left(\frac{\left[q_{\varepsilon}\right]}{\varepsilon}+\frac{\left[w_{\varepsilon}\right]}{\varepsilon}\right)-f\left(\frac{\left[w_{\varepsilon}\right]}{\varepsilon}\right)=\mathcal{B}_{k}^{\varepsilon}+\sigma^{\varepsilon} \nabla q_{\varepsilon} \cdot \nu_{\varepsilon}, \quad \text { on } \Gamma_{k}^{\varepsilon} \times(0, T),
$$

where

$$
\mathcal{B}_{k}^{\varepsilon}(t)=\frac{1}{\left|\Gamma_{k}^{\varepsilon}\right|} \int_{\Gamma_{k}^{\varepsilon}} f\left(\frac{\left[u_{\varepsilon}\right]}{\varepsilon}\right) \mathrm{d} \sigma-f\left(\frac{\left[w_{\varepsilon}\right]}{\varepsilon}\right) .
$$

In the following we exploit the fact that $\mathcal{B}_{k}$ depends on the time variable $t$ but not on the space variable $x$ on $\Gamma_{k}^{\varepsilon} \times(0, T)$.

Proposition 3.1. For every $\varepsilon>0$, let $q_{\varepsilon}$ be the function defined above. Then under the assumptions of Theorem 2.1 we have

$$
\frac{1}{\varepsilon} \int_{\Gamma^{\varepsilon}}\left[q_{\varepsilon}\right]^{2}(x, t) \mathrm{d} \sigma \leq \gamma \mathrm{e}^{-\beta t}, \quad \forall t>0
$$

where $\beta, \gamma>0$ are constants independent from $\varepsilon$.

Proof. We multiply equation (3.6)-(3.7) by $q_{\varepsilon}$ and integrate by parts in the space variable $x$. Taking into account equations (3.8), (3.13) and (3.11), we obtain

$$
\begin{aligned}
\int_{\Omega} \sigma^{\varepsilon}\left|\nabla q_{\varepsilon}\right|^{2} \mathrm{~d} x+\frac{\alpha}{\varepsilon} \int_{\Gamma^{\varepsilon}}\left[q_{\varepsilon, t}\right] & {\left[q_{\varepsilon}\right] \mathrm{d} \sigma } \\
& \quad+\int_{\Gamma^{\varepsilon}}\left(f\left(\frac{\left[q_{\varepsilon}\right]}{\varepsilon}+\frac{\left[w_{\varepsilon}\right]}{\varepsilon}\right)-f\left(\frac{\left[w_{\varepsilon}\right]}{\varepsilon}\right)\right)\left[q_{\varepsilon}\right] \mathrm{d} \sigma=0,
\end{aligned}
$$

where we used (3.12) and the fact that $\mathcal{B}_{k}$ is independent of $x$ on each $\Gamma_{k}^{\varepsilon} \times(0, T)$. Next we appeal to the bound from below of the first integral on the left hand side of (3.15) proved in (3.14) of [9], thus obtaining for a $\bar{\lambda}>0$ depending on the geometry of $\Gamma$ and on $\sigma$

$$
\begin{aligned}
\frac{\alpha}{\varepsilon} \bar{\lambda} \int_{\Gamma^{\varepsilon}}\left[q_{\varepsilon}\right]^{2} \mathrm{~d} \sigma+\frac{\alpha}{\varepsilon} \int_{\Gamma^{\varepsilon}}\left[q_{\varepsilon, t}\right]\left[q_{\varepsilon}\right] \mathrm{d} \sigma & \\
& +\int_{\Gamma^{\varepsilon}}\left(f\left(\frac{\left[q_{\varepsilon}\right]}{\varepsilon}+\frac{\left[w_{\varepsilon}\right]}{\varepsilon}\right)-f\left(\frac{\left[w_{\varepsilon}\right]}{\varepsilon}\right)\right)\left[q_{\varepsilon}\right] \mathrm{d} \sigma \leq 0 .
\end{aligned}
$$

If $f$ is monotone increasing the last integral in (3.16) is nonnegative and can be dropped; if this is not the case we have to assume $\bar{\lambda} \alpha>L_{-}$. Indeed, setting

$$
z_{\varepsilon}(t)=\int_{\Gamma^{\varepsilon}}\left[q_{\varepsilon}\right]^{2}(x, t) \mathrm{d} \sigma
$$


and taking into account (2.9), we infer from (3.16)

$$
\frac{\alpha}{2 \varepsilon} z_{\varepsilon}^{\prime}(t)+\frac{\alpha}{\varepsilon} \bar{\lambda} z_{\varepsilon}(t) \leq \frac{L_{-}}{\varepsilon} z_{\varepsilon}(t) .
$$

Inequality (3.17) implies

$$
\begin{array}{r}
z_{\varepsilon}(t) \leq z_{\varepsilon}(0) \mathrm{e}^{-2\left(\bar{\lambda}-L_{-} / \alpha\right) t}=\sum_{k \in \mathcal{I}}\left\{\int_{\Gamma_{k}^{\varepsilon}} S_{\varepsilon}^{2}(x) \mathrm{d} \sigma-\frac{1}{\left|\Gamma_{k}^{\varepsilon}\right|}\left(\int_{\Gamma_{k}^{\varepsilon}} S_{\varepsilon}(x) \mathrm{d} \sigma\right)^{2}\right\} \mathrm{e}^{-\beta t} \\
\leq \mathrm{e}^{-\beta t} \sum_{k \in \mathcal{I}} \int_{\Gamma_{k}^{\varepsilon}} S_{\varepsilon}^{2}(x) \mathrm{d} \sigma=\mathrm{e}^{-\beta t} \int_{\Gamma^{\varepsilon}} S_{\varepsilon}^{2}(x) \mathrm{d} \sigma \leq \gamma \varepsilon \mathrm{e}^{-\beta t},
\end{array}
$$

where $\beta=2\left(\bar{\lambda}-L_{-} / \alpha\right)$ and owing to assumption $(2.7) \gamma$ is a constant independent of $\varepsilon$. This proves the statement.

Remark 3.2. If $f$ is monotone increasing, $L_{-}$can be taken equal to zero, hence in (3.14) we have $\beta=2 \bar{\lambda}$. If in addition there exists $\kappa>0$ such that $f^{\prime}(s) \geq \kappa>0$ for every $s \in \boldsymbol{R}$, then equation (3.17) can be replaced with

$$
\frac{\alpha}{2 \varepsilon} z_{\varepsilon}^{\prime}(t)+\left(\frac{\alpha}{\varepsilon} \bar{\lambda}+\frac{\kappa}{\varepsilon}\right) z_{\varepsilon}(t) \leq 0
$$

yielding $\beta=2(\bar{\lambda}+\kappa / \alpha)$ in (3.14).

As a consequence of inequality (3.14) we can prove exponential decay to zero of $q_{\varepsilon}$ uniformly with respect to $\varepsilon>0$.

Proposition 3.3. For every $\varepsilon>0$, let $q_{\varepsilon}$ be the function defined above. Then

$$
\left\|q_{\varepsilon}(\cdot, t)\right\|_{L^{2}(\Omega)}^{2} \leq \gamma \mathrm{e}^{-\beta t} \quad \forall t>1
$$

where $\beta, \gamma>0$ are constants independent from $\varepsilon$.

Proof. By integrating (3.15) with respect to $t$ in the time interval $(\bar{t},+\infty)$ we get

$$
\int_{\bar{t}}^{+\infty} \int_{\Omega} \sigma^{\varepsilon}\left|\nabla q_{\varepsilon}\right|^{2} \mathrm{~d} x \mathrm{~d} t \leq \frac{L_{-}}{\varepsilon} \int_{\bar{t}}^{+\infty} \int_{\Gamma^{\varepsilon}}\left[q_{\varepsilon}\right]^{2} \mathrm{~d} \sigma \mathrm{d} t+\frac{\alpha}{2 \varepsilon} \int_{\Gamma^{\varepsilon}}\left[q_{\varepsilon}\right]^{2}(x, \bar{t}) \mathrm{d} \sigma, \quad \forall \bar{t}>0,
$$

where the first integral on the right hand side is finite owing to (3.14).

From (3.20) and (3.14) we get

$$
\int_{\bar{t}}^{+\infty} \int_{\Omega} \sigma^{\varepsilon}\left|\nabla q_{\varepsilon}\right|^{2} \mathrm{~d} x \mathrm{~d} t \leq \gamma \mathrm{e}^{-\beta \bar{t}}, \quad \forall \bar{t}>0 .
$$

Let $\bar{t}>1$ and $\psi \in \mathcal{C}^{\infty}([0,+\infty))$ be any increasing function such that $\psi(t)=0$ for $t \leq \bar{t}-1, \psi(t)=1$ for $t \geq \bar{t}$ and $\psi^{\prime}(t) \leq 2$. 
We multiply equation (3.6)-(3.7) by $\psi q_{\varepsilon, t}$ and integrate by parts with respect to $x$. Using (3.8), (3.11) and (3.13) we obtain

$$
\begin{aligned}
\int_{\Omega} \sigma^{\varepsilon} \nabla q_{\varepsilon} \nabla q_{\varepsilon, t} \psi(t) \mathrm{d} x & +\frac{\alpha}{\varepsilon} \int_{\Gamma^{\varepsilon}}\left[q_{\varepsilon, t}\right]^{2} \psi(t) \mathrm{d} \sigma \\
& +\int_{\Gamma^{\varepsilon}}\left(f\left(\frac{\left[q_{\varepsilon}\right]}{\varepsilon}+\frac{\left[w_{\varepsilon}\right]}{\varepsilon}\right)-f\left(\frac{\left[w_{\varepsilon}\right]}{\varepsilon}\right)\right)\left[q_{\varepsilon, t}\right] \psi(t) \mathrm{d} \sigma=0 .
\end{aligned}
$$

Here we again exploited the fact that $\mathcal{B}_{k}^{\varepsilon}$ depends only on $t$, and (3.12) as well. On integrating (3.22) over $[\bar{t}-1, \bar{t}]$ and taking into account assumption (2.8), we easily get

$$
\begin{aligned}
\int_{\Omega} \frac{\sigma^{\varepsilon}}{2}\left|\nabla q_{\varepsilon}(x, \bar{t})\right|^{2} \mathrm{~d} x & +\frac{\alpha}{\varepsilon} \int_{\bar{t}-1}^{\bar{t}} \int_{\Gamma^{\varepsilon}}\left[q_{\varepsilon, t}\right]^{2} \psi \mathrm{d} \sigma \mathrm{d} t \\
& \leq \frac{L}{\varepsilon} \int_{\bar{t}-1}^{\bar{t}} \int_{\Gamma^{\varepsilon}}\left|\left[q_{\varepsilon}\right]\right|\left|\left[q_{\varepsilon, t}\right]\right| \psi \mathrm{d} \sigma \mathrm{d} t+\int_{\bar{t}-1}^{\bar{t}} \int_{\Omega} \frac{\sigma^{\varepsilon}}{2}\left|\nabla q_{\varepsilon}\right|^{2} \psi^{\prime} \mathrm{d} x \mathrm{~d} t .
\end{aligned}
$$

Then by invoking (3.14) and (3.21) we estimate

$$
\begin{aligned}
& \int_{\Omega} \frac{\sigma^{\varepsilon}}{2}\left|\nabla q_{\varepsilon}(x, \bar{t})\right|^{2} \mathrm{~d} x+\frac{\alpha}{2 \varepsilon} \int_{\bar{t}-1}^{\bar{t}} \int_{\Gamma^{\varepsilon}}\left[q_{\varepsilon, t}\right]^{2} \psi \mathrm{d} \sigma \mathrm{d} t \\
& \leq \frac{L^{2}}{2 \alpha \varepsilon} \int_{\bar{t}-1}^{\bar{t}} \int_{\Gamma^{\varepsilon}}\left[q_{\varepsilon}\right]^{2} \mathrm{~d} \sigma \mathrm{d} t+\int_{\bar{t}-1}^{\bar{t}} \int_{\Omega} \sigma^{\varepsilon}\left|\nabla q_{\varepsilon}\right|^{2} \mathrm{~d} x \mathrm{~d} t \\
& \leq \frac{L^{2}}{2 \alpha \varepsilon} \int_{\bar{t}-1}^{+\infty} \int_{\Gamma^{\varepsilon}}\left[q_{\varepsilon}\right]^{2} \mathrm{~d} \sigma \mathrm{d} t+\int_{\bar{t}-1}^{+\infty} \int_{\Omega} \sigma^{\varepsilon}\left|\nabla q_{\varepsilon}\right|^{2} \mathrm{~d} x \mathrm{~d} t \leq \gamma \mathrm{e}^{-\beta(\bar{t}-1)}
\end{aligned}
$$

Collecting (3.14) and (3.24) we get

$$
\frac{1}{\varepsilon} \int_{\Gamma^{\varepsilon}}\left[q_{\varepsilon}(x, \bar{t})\right]^{2} \mathrm{~d} \sigma+\int_{\Omega}\left|\nabla q_{\varepsilon}(x, \bar{t})\right|^{2} \mathrm{~d} x \leq \gamma \mathrm{e}^{-\beta \bar{t}}, \quad \forall \bar{t}>1 .
$$

The last inequality, together with the Poincaré type inequality proved in [6, Lemma 7.1] immediately yields (3.19). 
In order to complete the proof of Theorem 2.1, recalling the definition of $w_{\varepsilon}$ we remark that

$$
\begin{aligned}
& \left\|w_{\varepsilon}(\cdot, t)\right\|_{L^{2}(\Omega)}^{2}=\sum_{k \in \mathcal{I}} \int_{\Omega_{1, k}^{\varepsilon}}\left(w_{\varepsilon}(x, t)\right)^{2} \mathrm{~d} x=\sum_{k \in \mathcal{I}} \frac{\left|\Omega_{1, k}^{\varepsilon}\right|}{\left|\Gamma_{k}^{\varepsilon}\right|^{2}}\left(\int_{\Gamma_{k}^{\varepsilon}}\left[u_{\varepsilon}(x, t)\right] \mathrm{d} \sigma\right)^{2} \\
& \quad \leq \gamma \frac{\varepsilon^{N}}{\varepsilon^{N-1}} \sum_{k \in \mathcal{I}} \int_{\Gamma_{k}^{\varepsilon}}\left[u_{\varepsilon}(x, t)\right]^{2} \mathrm{~d} \sigma=\gamma \varepsilon^{2}\left(\frac{1}{\varepsilon} \int_{\Gamma^{\varepsilon}}\left[u_{\varepsilon}(x, t)\right]^{2} \mathrm{~d} \sigma\right) \leq \gamma \varepsilon^{2} \mathrm{e}^{\frac{2 L_{-}}{\alpha} t},
\end{aligned}
$$

owing to the energy inequality (3.3). In turn, (3.26) and Proposition 3.3 imply for $u_{\varepsilon}=q_{\varepsilon}+w_{\varepsilon}$

$$
\left\|u_{\varepsilon}(\cdot, t)\right\|_{L^{2}(\Omega)}^{2} \leq 2\left\|q_{\varepsilon}(\cdot, t)\right\|_{L^{2}(\Omega)}^{2}+2\left\|w_{\varepsilon}(\cdot, t)\right\|_{L^{2}(\Omega)}^{2} \leq 2 \gamma\left(\mathrm{e}^{-\beta t}+\varepsilon^{2} \mathrm{e}^{\frac{2 L_{-}}{\alpha} t}\right) .
$$

Theorem 2.1 is proved.

Remark 3.4. We point out that, in the case where $f$ is assumed to be monotone increasing, inequality (3.27) reduces to

$$
\left\|u_{\varepsilon}(\cdot, t)\right\|_{L^{2}(\Omega)}^{2} \leq 2\left\|q_{\varepsilon}(\cdot, t)\right\|_{L^{2}(\Omega)}^{2}+2\left\|w_{\varepsilon}(\cdot, t)\right\|_{L^{2}(\Omega)}^{2} \leq 2 \gamma\left(\mathrm{e}^{-\beta t}+\varepsilon^{2}\right),
$$

where we used (3.2) instead of (3.3).

The non-vanishing asymptotic character of the spatial $L^{2}$-norm of $u_{\varepsilon}$ is due to the fact that, in general, the jump $\left[u_{\varepsilon}\right]$ has nonzero mean value over each $\Gamma_{k}^{\varepsilon}$. Estimate (3.28) is important in the homogenization limit $\varepsilon \rightarrow 0$. More precisely, under the assumptions of Theorem 2.1 in [12], we have that $u_{\varepsilon} \rightarrow u$ when $\varepsilon \rightarrow 0$, where $u$ denotes the homogenization limit function, and hence it follows from (3.28)

$$
\|u(\cdot, t)\|_{L^{2}(\Omega)}^{2} \leq \gamma \mathrm{e}^{-\beta t}, \quad \forall t>1 ;
$$

i.e., the exponential decay of the spatial $L^{2}$-norm of $u$. We note also that inequalities (3.28) and (3.29) correspond exactly to the results obtained in the linear case in [9, Theorem 2 and Corollary 3].

Remark 3.5. In the case where $f$ is not monotone increasing to the best of our knowledge the existence of a homogenization limit of $u_{\varepsilon}$ has not been proved yet. However, if such a limit $u$ indeed exists, it still satisfies (3.29) as a consequence of (3.27).

\section{Asymptotic Convergence to a periodic steady state for the $\varepsilon$-PROBLEM}

In this section we will assume that $\Psi$ is not identically zero and that $f$ fulfills also assumption (2.12). Our purpose here is to prove Theorem 2.2, that is essentially that for $\varepsilon>0$ fixed, the solution $u_{\varepsilon}$ of problem (2.1)-(2.6) converges to the periodic solution $u_{\varepsilon}^{\#}$ of (2.13)-(2.17) as $t \rightarrow+\infty$.

We begin by proving some regularity results for $u_{\varepsilon}$. 
Lemma 4.1. Let $\varepsilon>0$ and $u_{\varepsilon} \in L^{2}\left((0, T) ; \mathcal{X}^{1}\left(\Omega_{\varepsilon}\right)\right)$ be the solution of $(2.1)-(2.6)$. Then $u_{\varepsilon} \in \mathcal{C}^{0}\left((0, T] ; \mathcal{X}^{1}\left(\Omega_{\varepsilon}\right)\right)$, so that $\left[u_{\varepsilon}\right] \in \mathcal{C}^{0}\left((0, T] ; L^{2}\left(\Gamma^{\varepsilon}\right)\right)$ uniformly with respect to $\varepsilon$.

Remark 4.2. Indeed, a variant of the following proof jointly with Lemma 5 in [7] (providing a suitable estimate of $\nabla u_{\varepsilon}(0, \cdot)$ ), permits to prove that actually $\left[u_{\varepsilon}\right] \in$ $\mathcal{C}^{0}\left([0, T] ; L^{2}\left(\Gamma^{\varepsilon}\right)\right)$, but with a non-uniform bound of the norm with respect to $\varepsilon$.

Proof. Firstly, we note that the solution of (2.1)-(2.6) satisfies an energy inequality. In fact, multiplying equation $(2.1)-(2.2)$ by $u_{\varepsilon}-\Psi$, integrating by parts and using assumptions (2.12) and (2.10), we get

$$
\int_{0}^{T} \int_{\Omega} \sigma^{\varepsilon}\left|\nabla u_{\varepsilon}\right|^{2} \mathrm{~d} x \mathrm{~d} t+\frac{\alpha}{\varepsilon} \sup _{t \in(0, T)} \int_{\Gamma^{\varepsilon}}\left[u_{\varepsilon}\right]^{2}(x, t) \mathrm{d} \sigma \leq \gamma(T) .
$$

Now, let $\tau>0$ and $v^{\tau}:[0,+\infty) \rightarrow \boldsymbol{R}$ be a regular function such that $0 \leq v^{\tau} \leq 1$, $v^{\tau}(t)=1$, for $t \geq \tau, v^{\tau}(0)=0$. Multiplying (2.1)-(2.2) by $\left(u_{\varepsilon, t}-\Psi_{t}\right) v^{\tau}$, integrating by parts and using (2.8), (2.10) and (4.1), we get

$$
\begin{aligned}
\sup _{t \in(\tau, T)} & \int_{\Omega} \frac{\sigma^{\varepsilon}}{2}\left|\nabla u_{\varepsilon}(x, t)\right|^{2} \mathrm{~d} x+\frac{\alpha}{2 \varepsilon} \int_{\tau}^{T} \int_{\Gamma^{\varepsilon}}\left[u_{\varepsilon, t}\right]^{2} \mathrm{~d} \sigma \mathrm{d} t \\
& \leq \sup _{t \in(0, T)} \int_{\Omega} \frac{\sigma^{\varepsilon}}{2}\left|\nabla u_{\varepsilon}(x, t)\right|^{2} v^{\tau}(t) \mathrm{d} x+\frac{\alpha}{2 \varepsilon} \int_{0}^{T} \int_{\Gamma^{\varepsilon}}\left[u_{\varepsilon, t}\right]^{2} v^{\tau} \mathrm{d} \sigma \mathrm{d} t \leq \gamma(\tau, T) .
\end{aligned}
$$

Inequalities (4.1) and (4.2) imply that $\left[u_{\varepsilon}\right] \in \mathcal{C}^{0}\left((0, T] ; L^{2}\left(\Gamma^{\varepsilon}\right)\right)$. Let now $\widehat{v}^{\tau}$ : $[0,+\infty) \rightarrow \boldsymbol{R}$ be a function such that $0 \leq \widehat{v}^{\tau} \leq 1, \widehat{v}^{\tau}(t)=1$, for $t \geq 2 \tau, \widehat{v}^{\tau}(t)=0$, for $0 \leq t \leq \tau$. Differentiating formally with respect to $t(2.1)-(2.6)$, multiplying the first two equations thus obtained by $\left(u_{\varepsilon, t}-\Psi_{t}\right) \widehat{v}^{\tau}(t)$, and finally integrating by parts, we obtain

$$
\begin{aligned}
& \int_{2 \tau}^{T} \int_{\Omega} \sigma^{\varepsilon}\left|\nabla u_{\varepsilon, t}\right|^{2} \mathrm{~d} x \mathrm{~d} t+\frac{\alpha}{\varepsilon} \sup _{t \in(2 \tau, T)} \int_{\Gamma^{\varepsilon}}\left[u_{\varepsilon, t}\right]^{2}(x, t) \mathrm{d} \sigma \\
& \quad \leq \int_{0}^{T} \int_{\Omega} \sigma^{\varepsilon}\left|\nabla u_{\varepsilon, t}\right|^{2} \widehat{v}^{\tau}(t) \mathrm{d} x \mathrm{~d} t+\frac{\alpha}{\varepsilon} \sup _{t \in(0, T)} \int_{\Gamma^{\varepsilon}}\left[u_{\varepsilon, t}\right]^{2}(x, t) \widehat{v}^{\tau}(t) \mathrm{d} \sigma \leq \gamma(\tau, T),
\end{aligned}
$$

where we used also (4.2). Inequalities (4.2) and (4.3) imply $\nabla u_{\varepsilon} \in \mathcal{C}^{0}\left((0, T] ; L^{2}(\Omega)\right)$. This fact, jointly with Poincaré's inequality proved in [6, Lemma 7.1], implies that $u_{\varepsilon} \in \mathcal{C}^{0}\left((0, T] ; \mathcal{X}^{1}\left(\Omega_{\varepsilon}\right)\right)$. The statement is proved.

Remark 4.3. In fact Lemma 4.1 is still valid under the weaker assumptions $f \in \mathcal{C}^{1}(\boldsymbol{R})$, $f^{\prime} \geq 0$. 
Set $v_{\varepsilon}(x, t)=u_{\varepsilon}(x, t+1)-u_{\varepsilon}(x, t)$. Clearly $v_{\varepsilon}$ satisfies

$$
\begin{aligned}
-\operatorname{div}\left(\sigma^{\varepsilon} \nabla v_{\varepsilon}\right) & =0, & & \text { in }\left(\Omega_{1}^{\varepsilon} \cup \Omega_{2}^{\varepsilon}\right) \times(0,+\infty) ; \\
{\left[\sigma^{\varepsilon} \nabla v_{\varepsilon} \cdot \nu_{\varepsilon}\right] } & =0, & & \text { on } \Gamma^{\varepsilon} \times(0,+\infty) ; \\
\frac{\alpha}{\varepsilon} \frac{\partial}{\partial t}\left[v_{\varepsilon}\right]+g_{\varepsilon}(x, t) \frac{\left[v_{\varepsilon}\right]}{\varepsilon} & =\sigma^{\varepsilon} \nabla v_{\varepsilon} \cdot \nu_{\varepsilon}, & & \text { on } \Gamma^{\varepsilon} \times(0,+\infty) ; \\
{\left[v_{\varepsilon}\right](x, 0) } & =\left[u_{\varepsilon}(x, 1)\right]-S_{\varepsilon}(x)=: \widehat{S}_{\varepsilon}(x), & & \text { on } \Gamma^{\varepsilon} ; \\
v_{\varepsilon}(x) & =0, & & \text { on } \partial \Omega \times(0,+\infty),
\end{aligned}
$$

where

$$
g_{\varepsilon}(x, t):= \begin{cases}f^{\prime}\left(\frac{\left[u_{\varepsilon}\right]}{\varepsilon}(x, t)\right) & \text { if }\left[u_{\varepsilon}\right](x, t)=\left[u_{\varepsilon}\right](x, t+1), \\ \frac{f\left(\frac{\left[u_{\varepsilon}\right]}{\varepsilon}(x, t+1)\right)-f\left(\frac{\left[u_{\varepsilon}\right]}{\varepsilon}(x, t)\right)}{\frac{\left[u_{\varepsilon}\right]}{\varepsilon}(x, t+1)-\frac{\left[u_{\varepsilon}\right]}{\varepsilon}(x, t)} & \text { if }\left[u_{\varepsilon}\right](x, t) \neq\left[u_{\varepsilon}\right](x, t+1),\end{cases}
$$

so that $g_{\varepsilon}(x, t) \geq \kappa>0$ and $\widehat{S}_{\varepsilon}(x)$ still satisfies assumption (2.7) because of the energy inequality (4.1) satisfied by $u_{\varepsilon}$.

Proposition 4.4. For every $\varepsilon>0$, let $v_{\varepsilon}$ be the function defined above and satisfying problem (4.4)-(4.8). Then

$$
\frac{1}{\varepsilon} \int_{\Gamma^{\varepsilon}}\left[v_{\varepsilon}\right]^{2}(x, t) \mathrm{d} \sigma \leq \gamma \mathrm{e}^{-\beta t}, \quad \forall t>0,
$$

where $\beta=2 \kappa / \alpha$ and $\gamma>0$ are constants independent from $\varepsilon$.

Proof. Multiply equation (4.4) by $v_{\varepsilon}$ and integrate by parts with respect to $x$, thus obtaining

$$
\int_{\Omega} \sigma^{\varepsilon}\left|\nabla v_{\varepsilon}\right|^{2} \mathrm{~d} x+\frac{\alpha}{\varepsilon} \int_{\Gamma^{\varepsilon}}\left[v_{\varepsilon, t}\right]\left[v_{\varepsilon}\right] \mathrm{d} \sigma+\int_{\Gamma^{\varepsilon}} \frac{g_{\varepsilon}(x, t)}{\varepsilon}\left[v_{\varepsilon}\right]^{2} \mathrm{~d} \sigma=0,
$$

and hence

$$
\frac{\alpha}{\varepsilon} \int_{\Gamma^{\varepsilon}}\left[v_{\varepsilon, t}\right]\left[v_{\varepsilon}\right] \mathrm{d} \sigma+\frac{\kappa}{\varepsilon} \int_{\Gamma^{\varepsilon}}\left[v_{\varepsilon}\right]^{2} \mathrm{~d} \sigma \leq 0 .
$$

Even dropping the first integral in (4.10), we may make use here only of the property (2.12) of $f$ to get a result similar to the one in Proposition 3.1. In fact, setting

$$
z_{\varepsilon}(t)=\int_{\Gamma^{\varepsilon}}\left[v_{\varepsilon}\right]^{2}(t) \mathrm{d} \sigma
$$

we infer from (4.11)

$$
\frac{\alpha}{2 \varepsilon} z_{\varepsilon}^{\prime}(t)+\frac{\kappa}{\varepsilon} z(t) \leq 0
$$


whence the sought after estimate (4.9) in the form

$$
z_{\varepsilon}(t) \leq z_{\varepsilon}(0) \mathrm{e}^{-\frac{2 \kappa}{\alpha} t} \leq 2\left\{\int_{\Gamma^{\varepsilon}} S_{\varepsilon}^{2}(x) \mathrm{d} \sigma+\int_{\Gamma^{\varepsilon}}\left[u_{\varepsilon}\right]^{2}(x, 1)\right\} \mathrm{e}^{-\frac{2 \kappa}{\alpha} t} \leq \gamma \varepsilon \mathrm{e}^{-\beta t} .
$$

Indeed $\gamma$ is independent of $\varepsilon$ because of assumption (2.7) and of (4.1), and $\beta=$ $2 \kappa / \alpha$.

Remark 4.5. In this case, i.e., when we consider a nonhomogeneous boundary data, we cannot apply inequality (3.14) of [9] as we did in Section 3. Indeed the assumption that $\left[v_{\varepsilon}\right]$ has null mean average is not satisfied any longer and we cannot reduce to this case because of the nonlinearity of the problem, which forces the presence of the nonconstant term $g_{\varepsilon}$ in (4.6).

On the contrary, if the problem is linear, i.e. $f(s)=\kappa s$, we can set, without loss of generality, the initial mean value of $\left[v_{\varepsilon}\right]$ equal to zero on each component of $\Gamma^{\varepsilon}$, and this property is preserved for all positive times. Then we can apply (3.14) of [9] obtaining a version of (4.11) with $\kappa$ replaced by $\kappa+\bar{\lambda} \alpha$, where $\bar{\lambda}$ is as in the proof of Proposition 3.1. This implies that the estimates in Propositions 4.4 and 4.6 and Lemmas 4.8 and 4.10 hold with $\beta=2\left(\frac{\kappa}{\alpha}+\bar{\lambda}\right)$, so that in Theorem 2.2 we obtain the optimal decay which takes into account both the membrane and the bulk dissipation, as in the case of homogeneous boundary conditions (see Remark 3.2).

Proposition 4.6. Under the assumptions of Proposition 4.4 we have

$$
\left\|v_{\varepsilon}(\cdot, t)\right\|_{L^{2}(\Omega)}^{2} \leq \gamma \mathrm{e}^{-\beta t}, \quad \forall t>1
$$

where $\beta=2 \kappa / \alpha$ and $\gamma>0$ are constants independent from $\varepsilon$.

Proposition 4.6 is proved following the ideas in the proof of Proposition 3.3, but some modifications are necessary due to the presence of the term $g_{\varepsilon}$ in (4.6).

Proof. Firstly, let $\bar{t}>0$ and integrate (4.10) with respect to $t$ in the time interval $(\bar{t},+\infty)$, thus obtaining

$$
\int_{\bar{t}}^{+\infty} \int_{\Omega} \sigma^{\varepsilon}\left|\nabla v_{\varepsilon}\right|^{2} \mathrm{~d} x \mathrm{~d} t \leq \frac{\alpha}{2 \varepsilon} \int_{\Gamma^{\varepsilon}}\left[v_{\varepsilon}(x, \bar{t})\right]^{2} \mathrm{~d} \sigma
$$

where we have made use of (4.9) and we have dropped the positive term $\int_{\Gamma^{\varepsilon}} \frac{g_{\varepsilon}(x, t)}{\varepsilon}\left[v_{\varepsilon}\right]^{2} \mathrm{~d} \sigma \mathrm{d} t$. From (4.15), using again (4.9), we get

$$
\int_{\bar{t}}^{+\infty} \int_{\Omega} \sigma^{\varepsilon}\left|\nabla v_{\varepsilon}\right|^{2} \mathrm{~d} x \mathrm{~d} t \leq \gamma \mathrm{e}^{-\beta \bar{t}}, \quad \forall \bar{t}>0
$$

where $\gamma$ does not depend on $\varepsilon$. Let now $\bar{t}>1$ and $\psi \in C^{\infty}([0,+\infty))$ be an increasing function such that $\psi(t)=0$ for $t \leq \bar{t}-1, \psi(t)=1$ for $t \geq \bar{t}$ and $\psi^{\prime}(t) \leq 2$. Multiply equation (4.4) by $v_{\varepsilon, t} \psi$, integrate by parts with respect to $x$ and then integrate with 
respect to time $t \in[\bar{t}-1, \bar{t}]$, thus obtaining

$$
\begin{array}{r}
\int_{\bar{t}-1}^{\bar{t}} \int_{\Omega} \sigma^{\varepsilon} \nabla v_{\varepsilon} \nabla v_{\varepsilon, t} \psi(t) \mathrm{d} x \mathrm{~d} t+\frac{\alpha}{\varepsilon} \int_{\bar{t}-1}^{\bar{t}} \int_{\Gamma^{\varepsilon}}\left[v_{\varepsilon, t}\right]^{2} \psi(t) \mathrm{d} \sigma \mathrm{d} t \\
+\int_{\bar{t}-1} \int_{\Gamma^{\varepsilon}} \frac{g_{\varepsilon}(x, t)}{\varepsilon}\left[v_{\varepsilon, t}\right]\left[v_{\varepsilon}\right] \psi(t) \mathrm{d} \sigma \mathrm{d} t=0 .
\end{array}
$$

Now, taking into account that the function $f$ is assumed to be Lipschitz-continuous (i.e. $\left.\left|g_{\varepsilon}\right| \leq L\right)$ we get

$$
\begin{aligned}
\int_{\Omega} \frac{\sigma^{\varepsilon}}{2}\left|\nabla v_{\varepsilon}(x, \bar{t})\right|^{2} \mathrm{~d} x & +\frac{\alpha}{\varepsilon} \int_{\bar{t}-1}^{\bar{t}} \int_{\Gamma^{\varepsilon}}\left[v_{\varepsilon, t}\right]^{2} \psi(t) \mathrm{d} \sigma \mathrm{d} t \\
& \leq \frac{L}{\varepsilon} \int_{\bar{t}-1}^{\bar{t}} \int_{\Gamma^{\varepsilon}}\left|\left[v_{\varepsilon}\right]\right|\left|\left[v_{\varepsilon, t}\right]\right| \psi(t) \mathrm{d} \sigma \mathrm{d} t+\int_{\bar{t}-1}^{\bar{t}} \int_{\Omega} \frac{\sigma^{\varepsilon}}{2}\left|\nabla v_{\varepsilon}\right|^{2} \psi^{\prime}(t) \mathrm{d} x \mathrm{~d} t,
\end{aligned}
$$

which implies

$$
\begin{aligned}
& \int_{\Omega} \frac{\sigma^{\varepsilon}}{2}\left|\nabla v_{\varepsilon}(x, \bar{t})\right|^{2} \mathrm{~d} x+\frac{\alpha}{2 \varepsilon} \int_{\bar{t}-1}^{\bar{t}} \int_{\Gamma^{\varepsilon}}\left[v_{\varepsilon, t}\right]^{2} \psi(t) \mathrm{d} \sigma \mathrm{d} t \\
& \leq \frac{L^{2}}{2 \alpha \varepsilon} \int_{\bar{t}-1}^{+\infty} \int_{\Gamma^{\varepsilon}}\left[v_{\varepsilon}\right]^{2} \mathrm{~d} \sigma \mathrm{d} t+\int_{\bar{t}-1}^{+\infty} \int_{\Omega} \sigma^{\varepsilon}\left|\nabla v_{\varepsilon}\right|^{2} \mathrm{~d} x \mathrm{~d} t \leq \gamma \mathrm{e}^{-\beta(\bar{t}-1)}=\widetilde{\gamma} \mathrm{e}^{-\beta \bar{t}},
\end{aligned}
$$

where we used (4.9) and (4.16) and we set $\widetilde{\gamma}=\gamma \mathrm{e}^{\beta}$.

Putting together (4.9) and (4.19) we get

$$
\frac{1}{\varepsilon} \int_{\Gamma^{\varepsilon}}\left[v_{\varepsilon}(x, \bar{t})\right]^{2} \mathrm{~d} \sigma+\int_{\Omega}\left|\nabla v_{\varepsilon}(x, \bar{t})\right|^{2} \mathrm{~d} x \mathrm{~d} t \leq \gamma \mathrm{e}^{-\beta \bar{t}}, \quad \forall \bar{t}>1,
$$

where $\gamma$ is independent of $\varepsilon$. This last inequality, together with Poincare's inequality proved in [6, Lemma 7.1], gives the exponential decay of the spatial $L^{2}$-norm of $v_{\varepsilon}$ and concludes the proof.

Next for $\varepsilon>0$ and $n \in \boldsymbol{N}, n \geq 1$, we set $u_{\varepsilon, n}(x, t):=u_{\varepsilon}(x, t+n)$.

Remark 4.7. We stress the fact that, for every $T>0, u_{\varepsilon, n} \in \mathcal{C}^{0}\left([0, T], L^{2}(\Omega)\right),\left[u_{\varepsilon, n}\right] \in$ $\mathcal{C}^{0}\left([0, T], L^{2}\left(\Gamma^{\varepsilon}\right)\right), \nabla u_{\varepsilon, n} \in \mathcal{C}^{0}\left([0, T], L^{2}\left(\Omega_{i}^{\varepsilon}\right)\right), i=1,2$. This is a straightforward consequence of Lemma 4.1, since $n \geq 1$.

In our next Lemma, we will prove that $u_{\varepsilon, n}$ and $\nabla u_{\varepsilon, n}$ are Cauchy sequences in $\mathcal{C}^{0}\left([0,1], L^{2}\left(\Omega_{i}^{\varepsilon}\right)\right)$, while $\left[u_{\varepsilon, n}\right] / \sqrt{\varepsilon}$ is a Cauchy sequence in $\mathcal{C}^{0}\left([0,1], L^{2}\left(\Gamma^{\varepsilon}\right)\right)$. 
Lemma 4.8. Let $\varepsilon>0$ and $\left\{u_{\varepsilon, n}\right\}_{n \in N}$ be the sequence of functions defined above. Then under the assumptions of Proposition 4.4, with $\beta=2 \kappa / \alpha$,

$$
\begin{aligned}
\left\|u_{\varepsilon, m}-u_{\varepsilon, n}\right\|_{\mathcal{C}^{0}\left([0,1], L^{2}(\Omega)\right)} & \leq \gamma \mathrm{e}^{-\beta n / 2}, & & \text { for every } m>n ; \\
\left\|\nabla u_{\varepsilon, m}-\nabla u_{\varepsilon, n}\right\|_{\mathcal{C}^{0}\left([0,1], L^{2}\left(\Omega_{i}^{\varepsilon}\right)\right)} & \leq \gamma \mathrm{e}^{-\beta n / 2}, & & \text { for every } m>n ; i=1,2 ; \\
\frac{1}{\sqrt{\varepsilon}}\left\|\left[u_{\varepsilon, m}\right]-\left[u_{\varepsilon, n}\right]\right\|_{\mathcal{C}^{0}\left([0,1], L^{2}\left(\Gamma^{\varepsilon}\right)\right)} & \leq \gamma \mathrm{e}^{-\beta n / 2}, & & \text { for every } m>n .
\end{aligned}
$$

Proof. Assume $m>n$. Because of (4.14), we have

$$
\begin{aligned}
& \left\|u_{\varepsilon, m}-u_{\varepsilon, n}\right\|_{\mathcal{C}^{0}\left([0,1], L^{2}(\Omega)\right)}=\sup _{t \in[0,1]}\left\|u_{\varepsilon}(\cdot, t+m)-u_{\varepsilon}(\cdot, t+n)\right\|_{L^{2}(\Omega)} \\
& \leq \sum_{j=n}^{m-1} \sup _{t \in[0,1]}\left\|u_{\varepsilon}(\cdot, t+(j+1))-u_{\varepsilon}(\cdot, t+j)\right\|_{L^{2}(\Omega)} \leq \gamma \sum_{j=n}^{m-1} \mathrm{e}^{-\beta j / 2} \leq \gamma \mathrm{e}^{-\beta n / 2},
\end{aligned}
$$

whence (4.21) follows. Reasoning in the same way and using (4.20) instead of (4.14), we obtain (4.22) and (4.23).

Since $\left\{u_{\varepsilon, n}\right\}_{n \in N}$ is a Cauchy sequence, it converges in the function spaces appearing in (4.21)-(4.23). Let us denote by $\bar{u}_{\varepsilon} \in \mathcal{C}^{0}\left([0,1], \mathcal{X}^{1}\left(\Omega_{\varepsilon}\right)\right)$, with $\left[\bar{u}_{\varepsilon}\right] \in \mathcal{C}^{0}\left([0,1], L^{2}\left(\Gamma^{\varepsilon}\right)\right)$, the limit of the sequence $\left\{u_{\varepsilon, n}\right\}_{n \in N}$ and by $u_{\varepsilon}^{\#}$ the periodic extension of $\bar{u}_{\varepsilon}$ to the whole time axis $(-\infty,+\infty)$.

Remark 4.9. Obviously, the regularity of $\bar{u}_{\varepsilon}$ implies that $u_{\varepsilon}^{\#} \in \mathcal{C}^{0}\left(\boldsymbol{R}, \mathcal{X}^{1}\left(\Omega_{\varepsilon}\right)\right)$ and $\left[u_{\varepsilon}^{\#}\right] \in \mathcal{C}^{0}\left(\boldsymbol{R}, L^{2}\left(\Gamma^{\varepsilon}\right)\right)$; indeed, we observe that $u_{\varepsilon}(x, 1+n)=u_{\varepsilon, n}(x, 1) \rightarrow \bar{u}_{\varepsilon}(x, 1)$ and $u_{\varepsilon}(x, n+1)=u_{\varepsilon, n+1}(x, 0) \rightarrow \bar{u}_{\varepsilon}(x, 0)$; hence, $\bar{u}_{\varepsilon}(x, 1)=\bar{u}_{\varepsilon}(x, 0)$ and the same holds for $\nabla \bar{u}_{\varepsilon}(x, 1)=\nabla \bar{u}_{\varepsilon}(x, 0)$ and $\left[\bar{u}_{\varepsilon}\right](x, 1)=\left[\bar{u}_{\varepsilon}\right](x, 0)$.

We are now in the position to prove one of the main results of this section.

Lemma 4.10. Under the assumptions of Proposition 4.4, and for $u_{\varepsilon}$ and $u_{\varepsilon}^{\#}$ as above, (2.18)-(2.20) hold true.

Proof. Let $t>0$ and set $n=\llbracket t \rrbracket$, where for $t \in \boldsymbol{R}$ we denote by $\llbracket t \rrbracket$ the integer part of $t$; then $t \in[n, n+1)$ and we have

$$
\begin{aligned}
& \left\|u_{\varepsilon}(\cdot, t)-u_{\varepsilon}^{\#}(\cdot, t)\right\|_{L^{2}(\Omega)}^{2} \leq \sup _{\hat{t} \in[n, n+1]}\left\|u_{\varepsilon}(\cdot, \hat{t})-u_{\varepsilon}^{\#}(\cdot, \hat{t})\right\|_{L^{2}(\Omega)}^{2} \\
& =\sup _{\tilde{t} \in[0,1]}\left\|u_{\varepsilon}(\cdot, \widetilde{t}+n)-u_{\varepsilon}^{\#}(\cdot, \tilde{t}+n)\right\|_{L^{2}(\Omega)}^{2}=\sup _{\widetilde{t} \in[0,1]}\left\|u_{\varepsilon}(\cdot, \widetilde{t}+n)-u_{\varepsilon}^{\#}(\cdot, \widetilde{t})\right\|_{L^{2}(\Omega)}^{2} \\
& \quad=\sup _{\widetilde{t} \in[0,1]}\left\|u_{\varepsilon}(\cdot, \widetilde{t}+n)-\bar{u}_{\varepsilon}(\cdot, \widetilde{t})\right\|_{L^{2}(\Omega)}^{2}
\end{aligned}
$$

Therefore from (4.21) we get

$$
\left\|u_{\varepsilon}(\cdot, t)-u_{\varepsilon}^{\#}(\cdot, t)\right\|_{L^{2}(\Omega)}^{2} \leq \gamma \mathrm{e}^{-\beta n}=\gamma \mathrm{e}^{-\beta \llbracket t \rrbracket} \leq \gamma \mathrm{e}^{\beta} e^{-\beta t}=\widetilde{\gamma} \mathrm{e}^{-\beta t},
$$

for every $t>0$, where $\widetilde{\gamma}=\gamma \mathrm{e}^{\beta}$ is independent of $\varepsilon$. This proves (2.18).

Reasoning as before and using (4.22) and (4.23) we obtain (2.19) and (2.20). 
Remark 4.11. Lemma 4.10 implies the exponential asymptotic convergence to zero of the solution $u_{\varepsilon}$, in the case $\Psi \equiv 0$. However, we point out that this result does not include Theorem 2.1 and Remark 3.2. Indeed, on one hand those results hold true even dispensing with the coercivity assumption (2.12), at the unavoidable expense of not proving decay to zero even if $L_{-}=0$ as in the case considered in this Section: recall the term $\varepsilon^{2}$ on the right hand side of (2.11). On the other hand, they prove a rate of convergence $2(\bar{\lambda}+\kappa / \alpha)$ rather than $2 \kappa / \alpha$ as in Lemma 4.10.

Remark 4.12. Taking into account (4.1) and (4.2), by (2.18)-(2.20) we obtain for all $t \in[1,2]$

$$
\begin{aligned}
& \left\|u_{\varepsilon}^{\#}(\cdot, t)\right\|_{L^{2}(\Omega)}^{2} \leq \gamma \mathrm{e}^{-\beta}+\left\|u_{\varepsilon}(\cdot, t)\right\|_{L^{2}(\Omega)}^{2} \leq \gamma, \\
& \left\|\nabla u_{\varepsilon}^{\#}(\cdot, t)\right\|_{L^{2}\left(\Omega_{i}^{\varepsilon}\right)}^{2} \leq \gamma \mathrm{e}^{-\beta}+\left\|\nabla u_{\varepsilon}(\cdot, t)\right\|_{L^{2}\left(\Omega_{i}^{\varepsilon}\right)}^{2} \leq \gamma, \quad i=1,2, \\
& \frac{1}{\varepsilon}\left\|\left[u_{\varepsilon}^{\#}\right](\cdot, t)\right\|_{L^{2}\left(\Gamma^{\varepsilon}\right)}^{2} \leq \gamma \mathrm{e}^{-\beta}+\frac{1}{\varepsilon}\left\|\left[u_{\varepsilon}\right](\cdot, t)\right\|_{L^{2}\left(\Gamma^{\varepsilon}\right)}^{2} \leq \gamma .
\end{aligned}
$$

Here we choose $t \in[1,2]$ because, in principle, (2.18)-(2.20) have not been proved when $t \rightarrow 0$. This restriction is immaterial for the application of these estimates of the periodic function $u_{\varepsilon}^{\#}$ in Theorem 4.14 below.

In order to complete the proof of Theorem 2.2 we only need prove the claim that $u_{\varepsilon}^{\#}$ solves the periodic problem (2.13)-(2.17). This is the content of Theorem 4.14 below. Let us state in detail the following Definition.

Definition 4.13. A weak solution $u_{\varepsilon}^{\#}$ of the periodic problem (2.13)-(2.17) is a timeperiodic function $u_{\varepsilon}^{\#} \in \mathcal{C}^{0}\left(\boldsymbol{R} ; \mathcal{X}^{1}\left(\Omega_{\varepsilon}\right)\right),\left[u_{\varepsilon}^{\#}\right] \in \mathcal{C}^{0}\left(\boldsymbol{R} ; L^{2}\left(\Gamma^{\varepsilon}\right)\right)$, with integer period, satisfying (2.16) in the trace sense and, for any function $\phi \in \mathcal{C}^{1}\left(\overline{\Omega_{i}^{\varepsilon}} \times \boldsymbol{R}\right), i=1,2$, having compact support in $\Omega \times \boldsymbol{R}$,

$$
\int_{\boldsymbol{R}} \int_{\Omega} \sigma^{\varepsilon} \nabla u_{\varepsilon}^{\#} \nabla \phi \mathrm{d} x \mathrm{~d} t-\frac{\alpha}{\varepsilon} \int_{\boldsymbol{R}} \int_{\Gamma^{\varepsilon}}\left[u_{\varepsilon}^{\#}\right]\left[\phi_{t}\right] \mathrm{d} \sigma \mathrm{d} t+\int_{\boldsymbol{R}} \int_{\Gamma^{\varepsilon}} f\left(\frac{\left[u_{\varepsilon}^{\#}\right]}{\varepsilon}\right)[\phi] \mathrm{d} \sigma \mathrm{d} t=0 .
$$

Theorem 4.14. For every $\varepsilon>0$, let $u_{\varepsilon}^{\#}$ be the function defined above. Then $u_{\varepsilon}^{\#}$ is the unique weak solution of problem (2.13)-(2.17).

Proof. 1) Existence.

The regularity and periodicity of $u_{\varepsilon}^{\#}$ have been already proved. The boundary condition (2.16) is obvious from the definition of $u_{\varepsilon}^{\#}$. It is only left to prove (4.30).

To this purpose, let $\phi$ be a regular function with compact support in $\Omega \times \boldsymbol{R}^{+}$as in Definition 4.13. From the autonomous character of problem (2.1)-(2.6) and from the standard definition of its weak solution, we get for all large enough $k \in \boldsymbol{N}$

$$
\begin{aligned}
\int_{\boldsymbol{R}^{+}} \int_{\Omega} \sigma^{\varepsilon} \nabla u_{\varepsilon}(x, t+k) \nabla \phi(x, t) \mathrm{d} x \mathrm{~d} t & -\frac{\alpha}{\varepsilon} \int_{\boldsymbol{R}^{+}} \int_{\Gamma^{\varepsilon}}\left[u_{\varepsilon}(x, t+k)\right]\left[\phi_{t}(x, t)\right] \mathrm{d} \sigma \mathrm{d} t \\
& +\int_{\boldsymbol{R}^{+}} \int_{\Gamma^{\varepsilon}} f\left(\frac{\left[u_{\varepsilon}(x, t+k)\right]}{\varepsilon}\right)[\phi(x, t)] \mathrm{d} \sigma \mathrm{d} t=0 .
\end{aligned}
$$


The convergence results of Lemma 4.10 imply as $k \rightarrow \infty$

$$
\begin{aligned}
\int_{\boldsymbol{R}^{+}} \int_{\Omega} \sigma^{\varepsilon} \nabla u_{\varepsilon}^{\#}(x, t) \nabla \phi(x, t) \mathrm{d} x \mathrm{~d} t-\frac{\alpha}{\varepsilon} \int_{\boldsymbol{R}^{+}} & \int_{\Gamma^{\varepsilon}}\left[u_{\varepsilon}^{\#}(x, t)\right]\left[\phi_{t}(x, t)\right] \mathrm{d} \sigma \mathrm{d} t+ \\
& +\int_{\boldsymbol{R}^{+}} \int_{\Gamma^{\varepsilon}} f\left(\frac{\left[u_{\varepsilon}^{\#}(x, t)\right]}{\varepsilon}\right)[\phi(x, t)] \mathrm{d} \sigma \mathrm{d} t=0,
\end{aligned}
$$

which amounts to the sought after (4.30).

2) Uniqueness.

Firstly we note that $u_{\varepsilon}^{\#}$ satisfies an energy estimate similar to (4.1), because of (4.27)-(4.29). Moreover, let us assume that another periodic solution $u_{\varepsilon}^{*}$ exists, and let $s \in \boldsymbol{N}$ be its period. Note that simply by the regularity requirements placed on any weak solution, bounds like those in (4.27)-(4.29) actually hold for $u_{\varepsilon}^{*}$ too, over its period. The constant $\gamma$ there might depend on $\varepsilon$, but this is immaterial in the present argument, where $\varepsilon$ is fixed. By the same token, $u_{\varepsilon}^{*}$ too satisfies an energy estimate like (4.1).

Setting $w_{\varepsilon}^{\#}:=u_{\varepsilon}^{\#}-u_{\varepsilon}^{*}$, we have that $w_{\varepsilon}^{\#}$ solves (in a suitable sense)

$$
\begin{array}{rlrl}
-\operatorname{div}\left(\sigma^{\varepsilon} \nabla w_{\varepsilon}^{\#}\right) & =0, & \text { in }\left(\Omega_{1}^{\varepsilon} \cup \Omega_{2}^{\varepsilon}\right) \times(0,+\infty) ; \\
{\left[\sigma^{\varepsilon} \nabla w_{\varepsilon}^{\#} \cdot \nu_{\varepsilon}\right]} & =0, & & \text { on } \Gamma^{\varepsilon} \times(0,+\infty) ; \\
\frac{\alpha}{\varepsilon} \frac{\partial}{\partial t}\left[w_{\varepsilon}^{\#}\right]+g_{\varepsilon}(x, t) \frac{\left[w_{\varepsilon}^{\#}\right]}{\varepsilon} & =\sigma^{\varepsilon} \nabla w_{\varepsilon}^{\#} \cdot \nu_{\varepsilon}, & & \text { on } \Gamma^{\varepsilon} \times(0,+\infty) ; \\
w_{\varepsilon}^{\#}(x, t) & =0, & \text { on } \partial \Omega \times(0,+\infty) ; \\
{\left[w_{\varepsilon}^{\#}\right](x, 0)} & =\left[u_{\varepsilon}^{\#}\right](x, 0)-\left[u_{\varepsilon}^{*}\right](x, 0)=: \widetilde{S}_{\varepsilon}(x), & \text { on } \Gamma^{\varepsilon},
\end{array}
$$

where

$$
g_{\varepsilon}(x, t):= \begin{cases}f^{\prime}\left(\frac{\left[u_{\varepsilon}^{*}\right]}{\varepsilon}(x, t)\right) & \text { if }\left[u_{\varepsilon}^{*}\right](x, t)=\left[u_{\varepsilon}^{\#}\right](x, t), \\ \frac{f\left(\frac{\left[u_{\varepsilon}^{\#}\right]}{\varepsilon}(x, t)\right)-f\left(\frac{\left[u_{\varepsilon}^{*}\right]}{\varepsilon}(x, t)\right)}{\frac{\left[u_{\varepsilon}^{\#}\right]}{\varepsilon}(x, t)-\frac{\left[u_{\varepsilon}^{*}\right]}{\varepsilon}(x, t)} & \text { if }\left[u_{\varepsilon}^{*}\right](x, t) \neq\left[u_{\varepsilon}^{\#}\right](x, t),\end{cases}
$$

so that $g_{\varepsilon}(x, t) \geq \kappa>0$ and $\widetilde{S}_{\varepsilon}(x)$ still satisfies the second assumption in (2.7). Then we consider $w_{\varepsilon}^{\#}$ as a solution of an initial value boundary problem.

We proceed as we did above in order to get inequality (4.14), finally obtaining

$$
\left.\| w_{\varepsilon}^{\#}(\cdot, t)\right) \|_{L^{2}(\Omega)}^{2} \leq \gamma \mathrm{e}^{-\beta t} .
$$

However, $w_{\varepsilon}^{\#}$ is a periodic function; then (4.36) implies that it must be identically zero, hence $u_{\varepsilon}^{\#}$ and $u_{\varepsilon}^{*}$ coincide.

\section{EXPONENTIAL DECAY OF THE SOLUTION OF THE HOMOGENIZED PROBLEM}

The aim of this section is to prove a result similar to the one proved in the previous section, i.e., convergence of the solution to a periodic steady state, but here we 
consider the homogenized problem. To this purpose, let $\left(u, u^{1}\right)$ be the two-scale limit of the solution $u_{\varepsilon}$ of problem (2.1)-(2.6) when the initial data $S_{\varepsilon}$ satisfies the additional conditions (2.27)-(2.28).

We recall that, by [12, Theorem 2.1], the pair $\left(u, u^{1}\right)$ is the solution of problem (2.21)-(2.26) in the following sense.

Definition 5.1. A pair $\left(u, u^{1}\right) \in L^{2}\left((0, T) ; H^{1}(\Omega)\right) \times L^{2}\left(\Omega \times(0, T) ; \mathcal{X}_{\#}^{1}(Y)\right)$ is a weak solution of (2.21)-(2.26) if

$$
\begin{array}{r}
\int_{0}^{T} \int_{\Omega} \int_{Y} \sigma\left(\nabla u+\nabla_{y} u^{1}\right)\left(\nabla \phi+\nabla_{y} \Phi\right) \mathrm{d} x \mathrm{~d} y \mathrm{~d} t+\int_{0}^{T} \int_{\Omega} \int_{\Gamma} f\left(\left[u^{1}\right]\right)[\Phi] \mathrm{d} x \mathrm{~d} \sigma \mathrm{d} t \\
-\alpha \int_{0}^{T} \int_{\Omega} \int_{\Gamma}\left[u^{1}\right] \frac{\partial}{\partial t}[\Phi] \mathrm{d} x \mathrm{~d} \sigma \mathrm{d} t-\alpha \int_{\Omega} \int_{\Gamma}[\Phi] S_{1} \mathrm{~d} x \mathrm{~d} \sigma=0,
\end{array}
$$

for any function $\phi \in \mathcal{C}^{0}\left(0, T ; H_{0}^{1}(\Omega)\right)$, and any function $\Phi \in \mathcal{C}^{0}\left([0, T] ; L^{2}\left(\Omega ; \mathcal{X}_{\#}^{1}(Y)\right)\right)$ with $\left[\Phi_{t}\right] \in \mathcal{C}^{0}\left([0, T] ; L^{2}(\Omega \times \Gamma)\right)$ which vanishes at $t=T$.

Moreover we assume that $u$ satisfies the boundary condition on $\partial \Omega \times[0, T]$ in the trace sense and that $u^{1}$ is periodic in $Y$ and has zero mean value in $Y$ for every $(x, t) \in \Omega \times(0, T)$.

Lemma 5.2. Let $\left(u, u^{1}\right) \in L^{2}\left((0, T) ; H^{1}(\Omega)\right) \times L^{2}\left(\Omega \times(0, T), \mathcal{X}_{\#}^{1}(Y)\right)$ be the solution of $(2.21)-(2.26)$. Then $\left(u, u^{1}\right) \in \mathcal{C}^{0}\left((0, T] ; H^{1}(\Omega)\right) \times \mathcal{C}^{0}\left((0, T] ; L^{2}\left(\Omega ; \mathcal{X}_{\#}^{1}(Y)\right)\right)$ and $\left[u^{1}\right] \in \mathcal{C}^{0}\left((0, T] ; L^{2}(\Omega \times \Gamma)\right)$.

Proof. Firstly, we note that the solution of (2.21)-(2.26) satisfies an energy inequality. In fact, using $\left(u-\Psi, u^{1}\right)$ in (5.1) as test functions and integrating by parts (using assumption (2.8) and (2.10)), we get

$$
\int_{0}^{T} \int_{\Omega} \int_{Y} \sigma\left|\nabla u+\nabla_{y} u^{1}\right|^{2} \mathrm{~d} x \mathrm{~d} y \mathrm{~d} t+\alpha \sup _{t \in(0, T)} \int_{\Omega} \int_{\Gamma}\left[u^{1}(x, y, t)\right]^{2} \mathrm{~d} \sigma \mathrm{d} x \leq \gamma .
$$

Now, let $\tau>0$ and $v^{\tau}:[0,+\infty) \rightarrow \boldsymbol{R}$ be an increasing function such that $0 \leq v^{\tau} \leq 1$, $v^{\tau}(t)=1$, for $t \geq \tau, v^{\tau}(0)=0$. Using $\left(\left(u_{t}-\Psi_{t}\right) v^{\tau}, u_{t}^{1} v^{\tau}\right)$ as test function in (5.1), integrating by parts and using again assumption (2.8), (2.10) and (5.2), we get

$$
\begin{aligned}
& \sup _{t \in(\tau, T)} \int_{\Omega} \int_{Y} \frac{\sigma}{2}\left|\nabla u+\nabla_{y} u^{1}\right|^{2} \mathrm{~d} x \mathrm{~d} y+\frac{\alpha}{2} \int_{\tau}^{T} \int_{\Omega} \int_{\Gamma}\left[u_{t}^{1}\right]^{2} \mathrm{~d} \sigma \mathrm{d} x \mathrm{~d} t \leq \\
& \sup _{t \in(0, T)} \int_{\Omega} \int_{Y} \frac{\sigma}{2}\left|\nabla u+\nabla_{y} u^{1}\right|^{2} v^{\tau}(t) \mathrm{d} x \mathrm{~d} y+\frac{\alpha}{2} \int_{0}^{T} \int_{\Omega} \int_{\Gamma}\left[u_{t}^{1}\right]^{2} v^{\tau}(t) \mathrm{d} \sigma \mathrm{d} x \mathrm{~d} t \leq \gamma(\tau, T) .
\end{aligned}
$$

Inequalities (5.2) and (5.3) imply that $\left[u^{1}\right] \in \mathcal{C}^{0}\left((0, T] ; L^{2}(\Omega \times \Gamma)\right)$. Moreover, differentiating formally with respect to $t$ problem (2.21)-(2.26), multiplying equation (2.21) 
(differentiated with respect to $t$ ) by $\left(\left(u_{t}-\Psi_{t}\right) \widehat{v}^{\tau}, u_{t}^{1} \widehat{v}^{\tau}\right)$, where $\widehat{v}^{\tau}:[0,+\infty) \rightarrow \boldsymbol{R}$ is a function such that $0 \leq \widehat{v}^{\tau} \leq 1, \widehat{v}^{\tau}(t)=1$, for $t \geq 2 \tau, \widehat{v}^{\tau}(t)=0$, for $0 \leq t \leq \tau$, and finally integrating by parts, we obtain

$$
\begin{gathered}
\int_{2 \tau} \int_{\Omega} \int_{Y} \sigma\left|\nabla u_{t}+\nabla_{y} u_{t}^{1}\right|^{2} \mathrm{~d} x \mathrm{~d} y \mathrm{~d} t+\alpha \sup _{t \in(2 \tau, T)} \int_{\Omega} \int_{\Gamma}\left[u_{t}^{1}\right]^{2} \mathrm{~d} x \mathrm{~d} \sigma \\
\leq \int_{0}^{T} \int_{\Omega} \int_{Y} \sigma\left|\nabla u_{t}+\nabla_{y} u_{t}^{1}\right|^{2} \widehat{v}^{\tau}(t) \mathrm{d} x \mathrm{~d} y \mathrm{~d} t \\
+\alpha \sup _{t \in(0, T)} \int_{\Omega} \int_{\Gamma}\left[u_{t}^{1}\right]^{2} \widehat{v}^{\tau}(t) \mathrm{d} x \mathrm{~d} \sigma \leq \gamma(\tau, T)
\end{gathered}
$$

where we used assumption (2.8), (2.10), (5.2) and (5.3).

Using (5.2)-(5.4), we finally get

$$
\sup _{t \in(2 \tau, T)} \int_{\Omega}|\nabla u|^{2} \mathrm{~d} x+\sup _{t \in(2 \tau, T)} \int_{\Omega} \int_{Y}\left|\nabla_{y} u^{1}\right|^{2} \mathrm{~d} x \mathrm{~d} y \leq \gamma(\tau, T),
$$

and

$$
\int_{2 \tau}^{T} \int_{\Omega}\left|\nabla u_{t}\right|^{2} \mathrm{~d} x \mathrm{~d} t+\int_{2 \tau}^{T} \int_{\Omega} \int_{Y}\left|\nabla_{y} u_{t}^{1}\right|^{2} \mathrm{~d} x \mathrm{~d} y \mathrm{~d} t \leq \gamma(\tau, T),
$$

by calculations similar ot those in (5.12) below. Inequalities (5.5) and (5.6) imply $\left(\nabla u, \nabla_{y} u^{1}\right) \in \mathcal{C}^{0}\left((0, T] ; L^{2}(\Omega \times Y)\right)$. This fact, jointly with Poincaré's inequality proved in $\left[6\right.$, Lemma 7.1], yields that $\left(u, u^{1}\right) \in \mathcal{C}^{0}\left((0, T] ; H^{1}(\Omega)\right) \times \mathcal{C}^{0}\left((0, T] ; L^{2}\left(\Omega ; \mathcal{X}_{\#}^{1}(Y)\right)\right)$. The proof is concluded.

For later use, let us define

$$
\begin{aligned}
& \|\|\left(h, h^{1}\right)\|\|:=\|h\|_{\mathcal{C}^{0}\left([0,1] ; L^{2}(\Omega)\right)}+\|\nabla h\|_{\mathcal{C}^{0}\left([0,1] ; L^{2}(\Omega)\right)} \\
& \quad+\left\|h^{1}\right\|_{\mathcal{C}^{0}\left([0,1] ; L^{2}(\Omega \times Y)\right)}+\left\|\nabla_{y} h^{1}\right\|_{\mathcal{C}^{0}\left([0,1] ; L^{2}(\Omega \times Y)\right)}+\left\|\left[h^{1}\right]\right\|_{\mathcal{C}^{0}\left([0,1] ; L^{2}(\Omega \times \Gamma)\right)},
\end{aligned}
$$

where $\left(h, h^{1}\right) \in \mathcal{C}^{0}\left([0,1] ; H^{1}(\Omega)\right) \times \mathcal{C}^{0}\left([0,1] ; L^{2}\left(\Omega ; \mathcal{X}_{\#}^{1}(Y)\right)\right.$, and

$$
\|\|\left(\widetilde{h}, \widetilde{h}^{1}\right) \mid\|\|:=\|\widetilde{h}\|_{H^{1}(\Omega)}+\left\|\widetilde{h}^{1}\right\|_{L^{2}(\Omega \times Y)}+\left\|\nabla_{y} \widetilde{h}^{1}\right\|_{L^{2}(\Omega \times Y)}+\left\|\left[\widetilde{h}^{1}\right]\right\|_{L^{2}(\Omega \times \Gamma)},
$$

where $\left(\widetilde{h}, \widetilde{h}^{1}\right) \in H^{1}(\Omega) \times L^{2}\left(\Omega ; \mathcal{X}_{\#}^{1}(Y)\right)$.

As in Section 4 , we set $v(x, t)=u(x, t+1)-u(x, t)$ and $v^{1}(x, y, t)=u^{1}(x, y, t+$ $1)-u^{1}(x, y, t)$. Next we integrate (4.14) and (4.20) with respect to time in $(t, t+\eta)$, then we take the two-scale limit in the inequality thus obtained, where we use the two-scale lower semicontinuity of the norm, see $[2,3,20]$, and finally we differentiate 
with respect to $\eta$ the resulting integrals. We get for $\beta=2 \kappa / \alpha$

$$
\begin{aligned}
& \int_{\Omega} v^{2}(x, t) \mathrm{d} \sigma \leq \gamma \mathrm{e}^{-\beta t}, \\
& \int_{\Omega} \int_{\Gamma}\left[v^{1}\right]^{2}(x, y, t) \mathrm{d} \sigma \leq \gamma \mathrm{e}^{-\beta t}, \\
& \int_{\Omega} \int_{Y}\left|\nabla v(x, t)+\nabla_{y} v^{1}(x, y, t)\right|^{2} \mathrm{~d} x \mathrm{~d} y \leq \gamma \mathrm{e}^{-\beta t},
\end{aligned}
$$

for any $t>0$. From the previous inequalities we also get

$$
\begin{aligned}
& \int_{\Omega} \int_{Y}\left|\nabla_{y} v^{1}(x, y, t)\right|^{2} \mathrm{~d} y \mathrm{~d} x+\int_{\Omega}|\nabla v(x, t)|^{2} \mathrm{~d} x \\
\leq & \gamma \mathrm{e}^{-\beta t}-2 \int_{\Omega} \int_{Y} \nabla_{y} v^{1}(x, y, t) \nabla v(x, t) \mathrm{d} y \mathrm{~d} x \\
= & \gamma \mathrm{e}^{-\beta t}-2 \int_{\Omega} \nabla v(x, t)\left(\int_{Y} \nabla_{y} v^{1}(x, y, t) \mathrm{d} y\right) \mathrm{d} x \\
\leq & \gamma \mathrm{e}^{-\beta t}+2 \int_{\Omega}|\nabla v(x, t)|\left(\int_{\Gamma}\left|\left[v^{1}(x, y, t)\right]\right| \mathrm{d} \sigma\right) \mathrm{d} x \\
\leq & \gamma \mathrm{e}^{-\beta t}+\frac{1}{2|\Gamma|}|\Gamma| \int_{\Omega}|\nabla v(x, t)|^{2} \mathrm{~d} x+2|\Gamma| \int_{\Omega} \int_{\Gamma}\left[v^{1}(x, y, t)\right]^{2} \mathrm{~d} \sigma \mathrm{d} x \\
\leq & \gamma \mathrm{e}^{-\beta t}+\frac{1}{2} \int_{\Omega}|\nabla v(x, t)|^{2} \mathrm{~d} x+\gamma \mathrm{e}^{-\beta t},
\end{aligned}
$$

which implies

$$
\begin{aligned}
& \int_{\Omega}|\nabla v(x, t)|^{2} \mathrm{~d} x \leq \gamma \mathrm{e}^{-\beta t}, \\
& \int_{\Omega} \int_{Y}\left|\nabla_{y} v^{1}(x, y, t)\right|^{2} \mathrm{~d} y \mathrm{~d} x \leq \gamma \mathrm{e}^{-\beta t} .
\end{aligned}
$$

Finally, using Poincaré's inequality [7, Proposition 4], see also [32], and the fact that $v^{1}$ has zero mean value on $Y$ for every $t>0$, we have

$$
\int_{\Omega} \int_{Y}\left|v^{1}(x, y, t)\right|^{2} \mathrm{~d} y \mathrm{~d} x \leq \gamma \mathrm{e}^{-\beta t} .
$$

Now, as in Section 4 , for $n \in \boldsymbol{N}, n \geq 1$, we set $u_{n}(x, t):=u(x, t+n)$ and $u_{n}^{1}(x, t):=$ $u^{1}(x, t+n)$. In the next lemma, we will prove that $\left(u_{n}, u_{n}^{1}\right)$ and $\left(\nabla u_{n}, \nabla u_{n}^{1}\right)$ are 
Cauchy sequences in $\mathcal{C}^{0}\left([0,1] ; L^{2}(\Omega)\right) \times \mathcal{C}^{0}\left([0,1] ; L^{2}(\Omega \times Y)\right)$, and the same holds for $\left[u_{n}^{1}\right]$ in $\mathcal{C}^{0}\left([0,1] ; L^{2}(\Omega \times \Gamma)\right)$.

Lemma 5.3. Let $\left\{\left(u_{n}, u_{n}^{1}\right)\right\}_{n \in N}$ be the sequence of pairs defined above. Then for $\beta=2 \kappa / \alpha$

$$
\begin{array}{rlrl}
\left\|u_{m}-u_{n}\right\|_{\mathcal{C}^{0}\left([0,1] ; L^{2}(\Omega)\right)} & \leq \gamma \mathrm{e}^{-\beta n / 2}, & & \text { for every } m>n ; \\
\left\|\nabla u_{m}-\nabla u_{n}\right\|_{\mathcal{C}^{0}\left([0,1] ; L^{2}(\Omega)\right)} \leq \gamma \mathrm{e}^{-\beta n / 2}, & & \text { for every } m>n ; \\
\left\|u_{m}^{1}-u_{n}^{1}\right\|_{\mathcal{C}^{0}\left([0,1] ; L^{2}(\Omega \times Y)\right)} \leq \gamma \mathrm{e}^{-\beta n / 2}, & & \text { for every } m>n ; \\
\left\|\nabla_{y} u_{m}^{1}-\nabla_{y} u_{n}^{1}\right\|_{\mathcal{C}^{0}\left([0,1] ; L^{2}(\Omega \times Y)\right)} \leq \gamma \mathrm{e}^{-\beta n / 2}, & & \text { for every } m>n ; \\
\left\|\left[u_{m}\right]-\left[u_{n}\right]\right\|_{\mathcal{C}^{0}\left([0,1] ; L^{2}(\Omega \times \Gamma)\right)} \leq \gamma \mathrm{e}^{-\beta n / 2}, & & \text { for every } m>n .
\end{array}
$$

Proof. Assume that $m>n$. Proceeding as done in Lemma 4.8 and using (5.9), (5.10), (5.13)-(5.15), we get

$$
\left\|\left|\left(u_{m}, u_{m}^{1}\right)-\left(u_{n}, u_{n}^{1}\right)\right|\right\| \leq \sum_{k=n}^{m-1}\left\|\left|\left(u_{k+1}, u_{k+1}^{1}\right)-\left(u_{k}, u_{k}^{1}\right)\right|\right\| \leq \gamma \mathrm{e}^{-\beta n / 2},
$$

where $\||| \cdot||$ is defined in (5.7).

The previous lemma implies that there exists a pair of functions $\left(\bar{u}, \bar{u}^{1}\right) \in \mathcal{C}^{0}\left([0,1] ; H^{1}(\Omega)\right) \times$ $\mathcal{C}^{0}\left([0,1] ; L^{2}\left(\Omega ; \mathcal{X}_{\#}^{1}(Y)\right)\right.$ such that

$$
\left\|\left|\left(\bar{u}, \bar{u}^{1}\right)-\left(u_{n}, u_{n}^{1}\right)\right|\right\| \leq \gamma \mathrm{e}^{-\beta n / 2} .
$$

We now denote by $\left(u^{\#}, u^{1, \#}\right)$ the periodic extension of $\left(\bar{u}, \bar{u}^{1}\right)$ to the whole time interval $(-\infty,+\infty)$ and we prove the exponential asymptotic decay of $\left(u(x, t), u^{1}(x, t)\right)$ to $\left(u^{\#}(x, t), u^{1, \#}(x, t)\right)$ as $t \rightarrow+\infty$, in the sense specified in Lemma 5.5.

We can prove that $\left(u^{\#}, u^{1, \#}\right) \in \mathcal{C}_{\#}^{0}\left(\boldsymbol{R} ; H^{1}(\Omega)\right) \times \mathcal{C}_{\#}^{0}\left(\boldsymbol{R} ; L^{2}\left(\Omega ; \mathcal{X}_{\#}^{1}(Y)\right)\right.$, reasoning as in Remark 4.9.

Remark 5.4. Actually $\left[u_{t}^{1, \#}\right] \in L_{\#}^{2}\left(\boldsymbol{R} ; L^{2}(\Omega \times \Gamma)\right)$. Indeed, in estimate (5.3) the time integration domain $(\tau, T)$ on the left-hand side can be replaced with $(n, n+1), n \geq 2$, taking $\tau=1$, so that the constant $\gamma(\tau, T)$ on the right-hand side actually is uniform over $n$. This implies that

$$
\int_{0}^{1} \int_{\Omega} \int_{\Gamma}\left[u_{n t}^{1}(x, y, t)\right]^{2} \mathrm{~d} t \mathrm{~d} x \mathrm{~d} \sigma \leq \gamma
$$

with $\gamma$ independent of $n$. Passing to the weak limit for $n \rightarrow+\infty$ the assert follows.

Lemma 5.5. Let $\left(u, u^{1}\right)$ be the solution of problem (2.22)-(2.26). Then, there exists $\widetilde{\gamma}>0$ such that for $\beta=2 \kappa / \alpha$

$$
\left\|||\left(u(\cdot, t), u^{1}(\cdot, t)\right)-\left(u^{\#}(\cdot, t), u^{1, \#}(\cdot, t)\right)|\|| \mid \leq \widetilde{\gamma} \mathrm{e}^{-\beta t / 2},\right.
$$

for every $t>1$. 
Proof. Let $t>1$ and $n:=\|t\|$ so that, setting $\tau=t-n$, we have $\tau \in[0,1)$ and $t=\tau+n$. From (5.21) and recalling (5.7) and (5.8), we get

$$
\begin{aligned}
& \|\|\left|\left(u(\cdot, t), u^{1}(\cdot, t)\right)-\left(u^{\#}(\cdot, t), u^{1, \#}(\cdot, t)\right)\right| \||| \\
& =\left|\left\|\mid\left(u(\cdot, \tau+n), u^{1}(\cdot, \tau+n)\right)-\left(u^{\#}(\cdot, \tau+n), u^{1, \#}(\cdot, \tau+n)\right)\right\| \|\right. \\
& \leq \mid\left\|\left(u(\cdot, \cdot+n), u^{1}(\cdot, \cdot+n)\right)-\left(u^{\#}(\cdot, \cdot+n), u^{1, \#}(\cdot, \cdot+n)\right)\right\| \| \\
& =\left|\left\|\left(u_{n}, u_{n}^{1}\right)-\left(u^{\#}, u^{1, \#}\right)\left|\left\|=\left|\left\|\left(u_{n}, u_{n}^{1}\right)-\left(\bar{u}, \bar{u}^{1}\right) \mid\right\| \leq \gamma \mathrm{e}^{-\beta n / 2} \leq \widetilde{\gamma} \mathrm{e}^{-\beta t / 2},\right.\right.\right.\right.\right.
\end{aligned}
$$

where we use the definition of $\left(u_{n}, u_{n}^{1}\right)$, the 1-periodicity with respect to time of $\left(u^{\#}, u^{1, \#}\right)$ and finally the definition of $\left(u^{\#}, u^{1, \#}\right)$, which implies that $\left(u^{\#}(x, \tau), u^{1, \#}(x, \tau)\right)=$ $\left(\bar{u}(x, \tau), \bar{u}^{1}(x, \tau)\right)$, for a.e. $(x, \tau) \in \Omega \times(0,1)$. This concludes the proof.

Remark 5.6. It is worthwile observing that the technique developed here does not rely on the homogenization process and thus can be applied to a more general class of two-scale systems, provided we know estimates like (5.9)-(5.11). For example think of a system of type (2.21)-(2.26) where the diffusion matrices may be in principle unrelated to each other, and depending on the space variables. Also including sources would be of interest.

We recall that in the linear case $f(s)=s$ the homogenized problem may be formulated both as an equation with memory and as a two-scale problem of type (2.21)-(2.26). Since it is known that the asymptotic decay for the former is not trivial (see [22], [23], [24]) one can see that the same issue for the general two-scale problem is not trivial either. In this section we reduced the difficulty to the proof of estimates (5.9)-(5.11).

Definition 5.7. We say that $\left(\mathrm{v}^{\#}, \mathrm{v}^{1, \#}\right) \in \mathcal{C}_{\#}^{0}\left(\boldsymbol{R} ; H^{1}(\Omega)\right) \times \mathcal{C}_{\#}^{0}\left(\boldsymbol{R} ; L^{2}\left(\Omega ; \mathcal{X}_{\#}^{1}(Y)\right)\right.$ with $\left[\mathrm{v}_{t}^{1, \#}\right] \in L_{\#}^{2}\left(\boldsymbol{R} ; L^{2}(\Omega \times \Gamma)\right)$ is a weak solution of $(2.29)-(2.34)$ if

$$
\begin{aligned}
& \int_{\boldsymbol{R}} \int_{\Omega} \int_{Y} \sigma\left(\nabla \mathrm{v}^{\#}(x, t)\right.\left.+\nabla_{y} \mathrm{v}^{1, \#}(x, y, t)\right)\left(\nabla \phi(x, t)+\nabla_{y} \Phi(x, y, t)\right) \mathrm{d} x \mathrm{~d} y \mathrm{~d} t \\
&+\int_{\boldsymbol{R}} \int_{\Omega} \int_{\Gamma} f\left(\left[\mathrm{v}^{1, \#}(x, y, t)\right]\right)[\Phi(x, y, t)] \mathrm{d} x \mathrm{~d} \sigma \mathrm{d} t \\
&-\alpha \int_{\boldsymbol{R}} \int_{\Omega} \int_{\Gamma}\left[\mathrm{v}^{1, \#}(x, y, t)\right] \frac{\partial}{\partial t}[\Phi(x, y, t)] \mathrm{d} x \mathrm{~d} \sigma \mathrm{d} t=0
\end{aligned}
$$

for every $(\phi, \Phi) \in \mathcal{C}_{0}^{0}\left(\boldsymbol{R} ; H_{0}^{1}(\Omega)\right) \times \mathcal{C}_{0}^{0}\left(\boldsymbol{R} ; L^{2}\left(\Omega ; \mathcal{X}_{\#}^{1}(Y)\right),\left[\Phi_{t}\right] \in L^{2}\left(\boldsymbol{R} ; L^{2}(\Omega \times \Gamma)\right)\right.$ and $\mathrm{v}^{1, \#}$ has zero mean value in $Y$ for every $(x, t) \in \Omega \times \boldsymbol{R}$. Moreover, $\mathrm{v}^{\#}$ satisfies (2.34) in the trace sense and is time-periodic with integer period.

Inequality (5.22) implies, indeed, the desired asymptotic convergence result Theorem 2.5, once we prove that $\left(u^{\#}, u^{1, \#}\right)$ solves the system of equations $(2.29)-(2.34)$ in the sense of Definition 5.7.

Such a result is a straightforward consequence of inequality (5.22) which allows us to pass to the limit as $t \rightarrow+\infty$ in the system of equations (2.21)-(2.26), as stated in the following Lemma, which actually proves the existence part of Theorem 2.4. 
Lemma 5.8. Assume that $\left(u^{\#}, u^{1, \#}\right)$ is the pair of functions defined above; then it satisfies (2.29)-(2.34) in the weak sense of Definition 5.7.

Proof. Let us assume in the variational formulation (5.1) that $\phi$ and $\Phi$ have compact support in $\left(t_{1}, t_{2}\right)$, for an arbitrary choice of $t_{2}>t_{1}>0$. Hence, taking into account the fact that the system (2.21)-(2.26) is autonomous, we get

$$
\begin{gathered}
\int_{t_{1}}^{t_{2}} \int_{\Omega} \int_{Y} \sigma\left(\nabla u(x, \bar{t}+t)+\nabla_{y} u^{1}(x, y, \bar{t}+t)\right)\left(\nabla \phi(x, t)+\nabla_{y} \Phi(x, y, t)\right) \mathrm{d} x \mathrm{~d} y \mathrm{~d} t \\
+\int_{t_{1}} \int_{\Omega} \int_{\Gamma} f\left(\left[u^{1}(x, y, \bar{t}+t)\right]\right)[\Phi(x, y, t)] \mathrm{d} x \mathrm{~d} \sigma \mathrm{d} t \\
\quad-\alpha \int_{t_{1}}^{t_{2}} \int_{\Omega} \int_{\Gamma}\left[u^{1}(x, y, \bar{t}+t)\right] \frac{\partial}{\partial t}[\Phi(x, y, t)] \mathrm{d} x \mathrm{~d} \sigma \mathrm{d} t=0,
\end{gathered}
$$

for every $\bar{t}>1-t_{1}$. Moreover, as a consequence of (5.22), we have

$$
\begin{aligned}
\sup _{\tau \in\left(t_{1}, t_{2}\right)}\|\|\left\|\left(u(\cdot, \tau+\bar{t}), u^{1}(\cdot, \tau+\bar{t})\right)-\left(u^{\#}(\cdot, \tau+\bar{t}), u^{1, \#}(\cdot, \tau+\bar{t})\right)\right\| \| \\
\quad \leq \sup _{\tau \in\left(t_{1}, t_{2}\right)} \widetilde{\gamma} \mathrm{e}^{-\beta(\tau+\bar{t}) / 2} \leq \widetilde{\gamma} \mathrm{e}^{-\beta t_{1} / 2} \mathrm{e}^{-\beta \bar{t} / 2}=\gamma \mathrm{e}^{-\beta \bar{t} / 2},
\end{aligned}
$$

where $\gamma=\widetilde{\gamma} \mathrm{e}^{-\beta t_{1} / 2}$. Hence we can pass to the limit as $\bar{t} \rightarrow+\infty$ thus obtaining

$$
\begin{array}{r}
\int_{t_{1}} \int_{\Omega} \int_{Y} \sigma\left(\nabla u^{\#}(x, t)+\nabla_{y} u^{1, \#}(x, y, t)\right)\left(\nabla \phi(x, t)+\nabla_{y} \Phi(x, y, t)\right) \mathrm{d} x \mathrm{~d} y \mathrm{~d} t \\
+\int_{t_{1}} \int_{\Omega} \int_{\Gamma} f\left(\left[u^{1, \#}(x, y, t)\right]\right)[\Phi(x, y, t)] \mathrm{d} x \mathrm{~d} \sigma \mathrm{d} t \\
\quad-\alpha \int_{t_{1}}^{t_{2}} \int_{\Omega} \int_{\Gamma}\left[u^{1, \#}(x, y, t)\right] \frac{\partial}{\partial t}[\Phi(x, y, t)] \mathrm{d} x \mathrm{~d} \sigma \mathrm{d} t=0
\end{array}
$$

Equation (5.26) essentially is the standard variational formulation of (2.29)-(2.34), i.e. (5.23). Also as a consequence of the convergence in (5.22) we have that $u^{\#}$ satisfies the boundary condition on $\partial \Omega$ in the trace sense, and $u^{1, \#}$ is periodic in $Y$ and has zero mean value in $Y$ for every $(x, t) \in \Omega \times \boldsymbol{R}$.

Finally, we prove the uniqueness result.

Completion of the proof of Theorem 2.4. Assume that two solutions, $\left(u_{1}^{\#}, u_{1}^{1, \#}\right)$ and $\left(u_{2}^{\#}, u_{2}^{1, \#}\right)$, exist and denote with $s \in \boldsymbol{N}$ any common period of both. Setting 
$\left(v^{\#}, v^{1 \#}\right):=\left(u_{1}^{\#}, u_{1}^{1, \#}\right)-\left(u_{2}^{\#}, u_{2}^{1, \#}\right)$, from (5.26) by means of a standard approximation of periodic testing functions with compactly supported functions we get

$$
\iint_{0}^{s} \int_{\Omega} \sigma\left|\nabla v^{\#}+\nabla_{y} v^{1, \#}\right|^{2} \mathrm{~d} x \mathrm{~d} y \mathrm{~d} t+\int_{0}^{s} \int_{\Omega} \int_{\Gamma} \frac{f\left(\left[u_{1}^{1, \#}\right]\right)-f\left(\left[u_{2}^{1, \#}\right]\right)}{\left[u_{1}^{1, \#}\right]-\left[u_{2}^{1, \#}\right]}\left[v^{1, \#}\right]^{2} \mathrm{~d} x \mathrm{~d} \sigma \mathrm{d} t=0
$$

where we have set $\phi=v^{\#}$ and $\Phi=v^{1, \#}$ in the weak formulation (5.23).

Equation (5.27) implies (since $f^{\prime} \geq \kappa>0$ ) that

$$
\left[v^{1, \#}\right] \equiv 0, \quad \text { in } \Omega \times Y \times[0, s] .
$$

Using (5.27) and (5.28) and proceeding as in (5.12), we get that

$$
\int_{0}^{s} \int_{\Omega} \sigma\left|\nabla v^{\#}\right|^{2} \mathrm{~d} x \mathrm{~d} t+\int_{0}^{s} \int_{\Omega} \int_{Y} \sigma\left|\nabla_{y} v^{1, \#}\right|^{2} \mathrm{~d} x \mathrm{~d} y \mathrm{~d} t=0 .
$$

The first integral of (5.29), together with the fact that $v^{\#}$ satisfies homogeneous boundary condition on $\partial \Omega \times[0, s]$, implies

$$
v^{\#}=0, \quad \text { in } \Omega \times[0, s] .
$$

Finally from (5.29), together with (5.28) and the fact that $v^{1, \#}$ has zero mean value, we have that

$$
v^{1, \#}=0, \quad \text { in } \Omega \times Y \times[0, s] .
$$

Uniqueness of the periodic solution of the homogenized problem is thus proved.

\section{REFERENCES}

[1] A. Abenavoli, M. Di Francesco, I. Schroeder, S. Epimashko, S. Gazzarrini, U. Hansen, G. Thiel, and A. Moroni. Fast and slow gating are inherent properties of the pore module of the $\mathrm{K}^{+}$ channel Kcv. The Journal of General Physiology, 134:219-229, 2009.

[2] G. Allaire. Homogenization and two-scale convergence. SIAM J. Math. Anal., 23:1482-1518, 1992.

[3] G. Allaire, A. Damlamian, and U. Hornung. Two-scale convergence on periodic surfaces and applications. Proceedings of the International Conference on Mathematical Modelling of Flow through Porous Media, 15-25, 1995.

[4] M. Amar, D. Andreucci, P. Bisegna, and R. Gianni. Homogenization limit for electrical conduction in biological tissues in the radio-frequency range. Comptes Rendus Mecanique, 331:503-508, 2003. Elsevier.

[5] M. Amar, D. Andreucci, P. Bisegna, and R. Gianni. An elliptic equation with history. C. $R$. Acad. Sci. Paris, Ser. I, 338:595-598, 2004. Elsevier.

[6] M. Amar, D. Andreucci, P. Bisegna, and R. Gianni. Evolution and memory effects in the homogenization limit for electrical conduction in biological tissues. Mathematical Models and Methods in Applied Sciences, 14:1261-1295, 2004. World Scientific.

[7] M. Amar, D. Andreucci, P. Bisegna, and R. Gianni. Existence and uniqueness for an elliptic problem with evolution arising in electrodynamics. Nonlinear Analysis Real World Applications, 6:367-380, 2005. Elsevier.

[8] M. Amar, D. Andreucci, P. Bisegna, and R. Gianni. On a hierarchy of models for electrical conduction in biological tissues. Mathematical Methods in the Applied Sciences, 29:767-787, 2006. 
[9] M. Amar, D. Andreucci, P. Bisegna, and R. Gianni. Exponential asymptotic stability for an elliptic equation with memory arising in electrical conduction in biological tissues. Euro. Jnl. of Applied Mathematics, 20:431-459, 2009.

[10] M. Amar, D. Andreucci, P. Bisegna, and R. Gianni. Stability and memory effects in a homogenized model governing the electrical conduction in biological tissues. J. Mechanics of Material and Structures, (2) 4:211-223, 2009.

[11] M. Amar, D. Andreucci, P. Bisegna, and R. Gianni. Homogenization limit and asymptotic decay for electrical conduction in biological tissues in the high radiofrequency range. Communications on Pure and Applied Analysis, (5) 9:1131-1160, 2010.

[12] M. Amar, D. Andreucci, P. Bisegna, and R. Gianni. A hierarchy of models for the electrical conduction in biological tissues via two-scale convergence: The nonlinear case. Differential and Integral Equations, (9-10) 26:885-912, 2013.

[13] M. Amar, D. Andreucci, and R. Gianni. Asymptotic decay under nonlinear and noncoercive dissipative effects for electrical conduction in biological tissues. In preparation.

[14] G. Beeler and H. Reuter. Reconstruction of the action potential of ventricular myocardial fibres. J. Physiol., 268(1):177-210, 1977.

[15] A. Bensoussan, J. L. Lions, and G. Papanicolaou. Asymptotic Analysis for Periodic Structures. North Holland, Amsterdam, 1978.

[16] P. Bisegna, G. Caruso, and F. Lebon. Bioelectrical impedance analysis: a matter of homogenization of composites with imperfect interfaces. In G. Augusti, editor, Proceedings 15th AIMETA Congress of Theoretical and Applied Mechanics. 2001.

[17] G. Bouchitté and M. Bellieud. Homogenization of a soft elastic material reinforced by fibers. Asymptotic Analysis, 32:153-183, 2002.

[18] J. D. Bronzino. The Biomedical Engineering Handbook. CRC Press, 1999.

[19] D. Cioranescu and P. Donato. An Introduction to Homogenization. Oxford University Press, New York, 1999.

[20] G. Clark and L. Packer. Two-scale homogenization of implicit degenerate evolution equations. Journal of Mathematical Analysis and Applications, 214:420-438, 1997.

[21] A. De Lorenzo, A. Andreoli, J. Matthie, and P. Withers. Predicting body cell mass with bioimpedence by using theoretical methods: a technological review. J. Appl. Physiol., 82:15421558, 1997.

[22] M. Fabrizio and B. Lazzari. Sulla stabilità di un sistema viscoelastico lineare. In Acc. Naz. Lincei, Tavola rotonda sul tema: Continui con Memoria. Roma, 1992.

[23] M. Fabrizio and A. Morro. Viscoelastic relaxation functions compatible with thermodynamics. Journal of Elasticity, 19:63-75, 1988.

[24] G. Fichera. Avere una memoria tenace crea gravi problemi. Arch. Ration. Mech. Anal., 70:101$112,1979$.

[25] K. R. Foster and H. P. Schwan. Dielectric properties of tissues and biological materials: a critical review. Critical Reviews in Biomedical Engineering, 17:25-104, 1989.

[26] C. Giorgi, M. G. Naso, and V. Pata. Exponential stability in linear heat conduction with memory: a semigroup approach. Commun. Appl. Anal., 5(1):121-133, 2001.

[27] C. Giorgi, M. G. Naso, and V. Pata. Energy decay of electromagnetic systems with memory. Math. Models Methods Appl. Sci., 15(10):1489-1502, 2005.

[28] H.-K. Hummel. Homogenization for heat transfer in polycrystals with interfacial resistances. Appl. Anal., 75:403-424, 2000.

[29] E. R. Kandell, J. H. Schwartz, and T. M. Jessell, editors. Principles of Neural Science. McGrawHill, New York, 2000. Fourth edition.

[30] W. Krassowska and J. C. Neu. Homogenization of syncytial tissues. Critical Reviews in Biomedical Engineering, 21:137-199, 1993.

[31] F. Lene and D. Leguillon. Étude de l'influence d'un glissement entre les constituants d'un matériau composite sur ses coefficients de comportement effectifs. Journal de Mécanique, 20:509-536, 1981. 
[32] R. Lipton. Heat conduction in fine scale mixtures with interfacial contact resistance. SIAM Journal of Applied Mathematics, 58:55-72, 1998.

[33] J. Malmivuo and R. Plonsey. Bioelectromagnetism. Oxford University Press, Oxford, 1995.

[34] G. Nguetseng. A general convergence result for a functional related to the theory of homogenization. SIAM J. Math. Anal., 20:608-623, 1989.

[35] E. Sánchez-Palencia. Non Homogeneous Media and Vibration Theory, volume 127 of Lecture notes in physics. Springer Verlag, 1980.

[36] C. Timofte. Multiscale analysis of diffusion processes in composite media. Computers and Mathematics with Applications, 66:1573-1580, 2013. 